\title{
Polygonal Areas in Regular and Distorted Lattices
}

\author{
DIMITRIOS KODOKOSTAS
}

\section{Distorted Lattices, Long Determinants and Statement of the Main Results}

Some sixteen or seventeen years ago as a youngster I've heard of a beautiful exercise claiming that if we divide each side of a convex quadrilateral in $2^{n}$ equal parts and join the corresponding division points of opposite sides by line segments, then by colouring the small quadrilaterals formed alternatively as black and white, the total black area equals the total white area! (Fig. 1a).

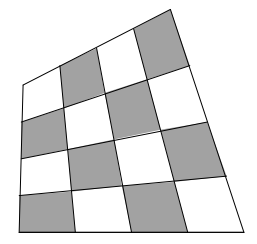

a

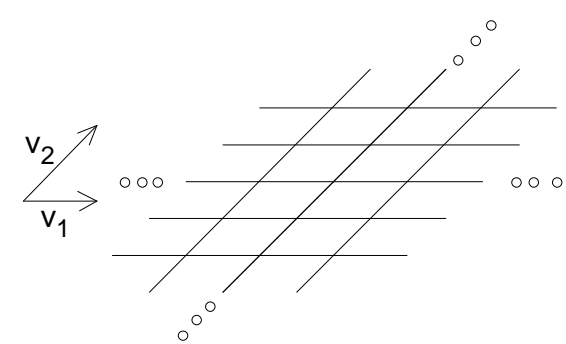

b

FIGURE 1

Recently, reflecting once again on the same problem I attempted a rigorous proof and I fell upon a rain of surprises. It is the goal of this article to share some of these surprises.

In the current section we will state the main results and we will develop the necessary notation and terminology for a concise exposition. Some technical details necessary for the proofs are delayed 
to Section 2 and the proofs themselves are postponed to Section 3. Section 4 contains some corollaries, facts, conjectures, challenges and exercises.

The most important result is by far Theorem 1 along with its Corollary.

So let us start with the results. First we prove

Proposition. Let $A B C D$ be a convex quadrilateral. If we divide each one of the sides $A B, D C$ into $m$ equal parts, and each of the sides $A D, B C$ into $n$ equal parts $\left(m, n \in \mathbb{N}^{*}\right)$, then by joining the corresponding division points of opposite sides by line segments, each of these segments from $A B$ to $D C$ is divided in $n$ equal parts, while each of these segments from $A D$ to $B C$ is divided in $m$ equal parts (Fig. 4c).

Then as an easy consequence we provide a proof of a generalization of the opening exercise to this article (cf. Corollary 1 in Section 3).

The above proposition is notable since it leads us to defining the notion of distorted lattices:

Recall that according to one of many equivalent definitions, a usual lattice in the plane is a set of vectors of the form $t_{1} \vec{v}_{1}+t_{2} \vec{v}_{2}$ where $\vec{v}_{1}, \vec{v}_{2}$ are linearly independent plane vectors and $t_{1}, t_{2} \in \mathbb{Z}$ (for example cf. [3]). Instead of vectors, we can equivalently think of the points they represent in the plane. Naturally, one cannot resist dissecting the plane in the parallelograms these points suggest by drawing the set of lines $H=\left\{t \vec{v}_{1}+t_{2} \vec{v}_{2}, t \in \mathbb{R}\right.$ and $t_{2}=$ constant in $\mathbb{Z}\}$, and the set of lines $V=\left\{t_{1} \vec{v}_{1}+t \vec{v}_{2}, t \in \mathbb{R}\right.$ and $t_{1}=$ constant in $\mathbb{Z}\}$ (Fig. 1b). Often, a lattice is defined as a set of lines of the above two forms. Each such line is called a lattice line and their intersection points are called lattice points.

Observe that all lattice lines in $H$ are parallel to each other, as are to each other all lattice lines in $V$. Observe also that on each lattice line the distance of any two successive lattice points is constant.

Trying to generalize the notion of a lattice we can ask for a system of two sets of lines $H, V$ in the plane so that not all lines in any of $H, V$ are necessarily parallel to each other. Of course we should at least ask for the property that on any line, any two consecutive intersection points with lines of the other set have a constant distance. It is clear that we cannot ask for this distance to remain the same for all lines of the same set. Unfortunately it is also clear that if the lines of either of these sets are not all parallel to each other then 
a large number of unpleasant facts occurs. So if we insist to retain the possibility that members of any one of the two families are not all parallel to each other, it is more convenient to drop the use of lines and instead introduce the use of line segments according to the following

Definition 1. A distorted (or generalized) plane lattice, consists of two sets of line segments, so that any two segments of the same set do not intersect, any two segments of different sets intersect, and the intersection points on each segment divide it into equal parts. We also ask that the endpoints of each segment are intersection points.

Examples of distorted lattices are given in Figure 2.

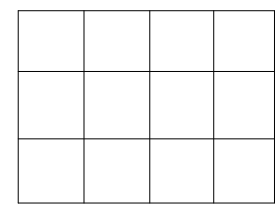

a

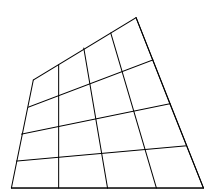

b

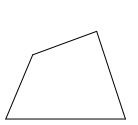

C

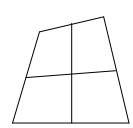

d

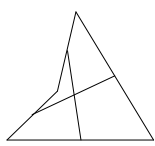

e

FiguRE 2

We shall call the segments belonging to one of the sets horizontal, and to the other perpendicular (with no preference on the assignments), and all of them as lattice segments. The intersection points will be called lattice points. The segments into which each lattice segment is divided by the intersection points will be called lattice sub-segments. Clearly any two horizontal segments are divided in the same number of sub-segments say $m$, and similarly any two vertical segments are also divided in the same number of sub-segments, say $n\left(m, n \in \mathbb{N}^{*}\right)$. Such a lattice will be called an $\{m, n\}$ distorted lattice and it will be denoted as $\mathbf{L}\{\mathbf{m}, \mathbf{n}\}$.

The last condition in the definition was given in order to avoid calling lattices such troublesome cases as those in Figure 3. Even with the provisions of the definition, "pathological" cases of lattices do occur, like the one in Figure 4a which is a $\{2,4\}$ distorted lattice. Conjecture 1a in Section 4 states that for $m \neq 1 \neq n$ such pathologies do not occur. We shall call lattices $L\{m, n\}$ with $m \neq 1 \neq n$ as non trivial whereas the rest as trivial. An example of a trivial distorted 
lattice is given in Figure 4b. The most trivial lattice is $L\{1,1\}$ which is nonhing else than a simple quadrilateral with horizontal segments its two opposite sides and vertical segments its other two sides (Fig. 2c).

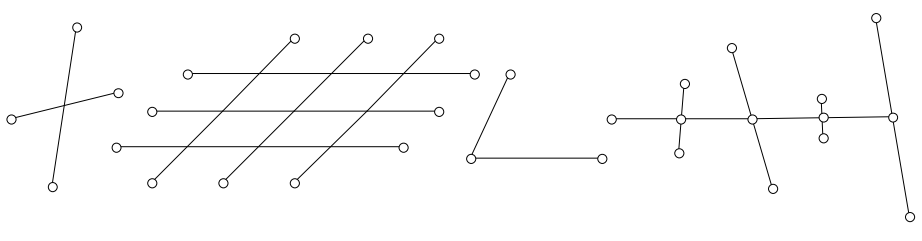

FiguRE 3

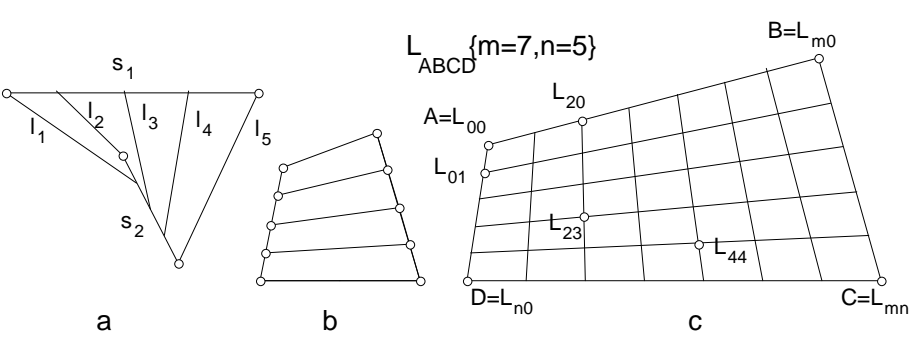

FiguRE 4

Finally let us call genuine distorted lattices those in which the horizontal segments are not all parallel to each other or the vertical segments are not all parallel to each other. All other distorted lattices will be called regular or usual. Regular lattices are just finite restrictions of the usual infinite lattices. For this reason we can call the usual infinite lattices as infinite regular lattices as well.

Note that Proposition 1 assures the existence of genuine distorted lattices $L\{m, n\}$ for no matter how large $m, n \in \mathbb{N}$.

In what follows we shall mostly be interested in distorted lattices arising from convex quadrilaterals in the way described in Proposition 1. In Section 4 we show that analogues of Proposition 1 hold in some cases for non-convex quadrilaterals $A B C D$ as well. The quadrilateral giving rise in such a way to a lattice will be called the associated quadrilateral to the lattice; the lattice itself is going 
to be called a convex or non-convex distorted lattice according to the nature of its associated quadrilateral, and it will be denoted as $\mathbf{L}_{A B C D}\{\mathbf{m}, \mathbf{n}\}$. All other lattices can be appropriately called as pathological distorted lattices. It is clear that all convex and some non-convex distorted lattices are divided by the lattice segments into small quadrilaterals which we shall call lattice quadrilaterals.

From now on and until further notice, all lattices will be convex distorted lattices but not necessarily non trivial or non genuine. The characterization "distorted"will be frequently dropped.

It is useful to fix notation and a universal picture in our minds for all convex quadrilaterals as well as for all distorted lattices which they produce. So let $A B C D$ be any convex quadrilateral. Without any loss of generality we will assume that for its interior angles hold $\widehat{B}+\widehat{C} \leq 180^{\circ}, \widehat{C}+\widehat{D} \leq 180^{\circ}$ (Fig. 5a). Such an assumption is permitted as is shown in Section 2. The first condition equivalently means that the half-lines $B A, C D$ intersect (or they are parallel and lie) at the same half-plane with respect to the line $B C$ at which the line segment $A D$ lies. Similarly for the second condition. We also arrange so that the orientation $(A, B, C, D)$ of $A B C D$ coincides with the clockwise (i.e. the negative) orientation of the plane. We shall say that such an $A B C D$ is in a nicely arranged position. We are going to place $D C$ in an as possible horizontal position with respect to our point of view. So $A B C D$ should look like in Figure 4c. We agree to consider as horizontal segments of any distorted lattice with associated quadrilateral $A B C D$ those lying at the same family with $A B, C D$. We shall call $A B$ as top and $C D$ as bottom horizontal segment. Similarly, $A D$ will be called left and $B C$ right vertical segment.

It is also convenient to assign to each lattice point of a nicely arranged lattice $L_{A B C D}\{m, n\}$, a pair of coordinates similar to the usual rectangular coordinates; only now, point $(a, b)$ with $0 \leq a \leq$ $m, 0 \leq b \leq n$ will mean the intersection point of the $(a+1)$ th vertical segment counting from left to right, with the $(b+1)$ th horizontal segment counting from top to bottom. Frequently this point will be denoted as $L_{a b}$ (Fig. 4c).

Our next and most important result refers to a way of calculating the area of any simple closed polygon with vertices in some lattice points of a (nicely arranged) convex distorted lattice $L_{A B C D}\{m, n\}$, 
in terms of the coordinates of its vertices. We shall call such polygons as lattice polygons. For a simple polygon $A_{1} A_{2} \ldots A_{k}$ we will be denoting its area by $\left(A_{1} A_{2} \ldots A_{k}\right)$.

We are not going to define a unit area and so we necessarily have to calculate the areas comparing them to a substitute of the unit area. Note that as the lattice quadrilaterals become smaller as we move to the top or to the left, the smallest one is the quadrilateral in the upper left corner. Since the four triangles in which this smallest quadrilateral is partitioned by its two diagonals are also lattice polygons, it is conceivable that the area of any other lattice polygon can be calculated in terms of the areas of these four triangles. It turns out that nice area calculations can be made in terms of the area $E$ of only the smallest triangle in the top left corner, but also with the help of the areas $E_{v}, E_{h}$ of two other triangles associated to the top left quadrilateral. To understand how these two triangles first make their appearance, we provide below the main lemma used for the calculations. The lemma refers to a very trivial lattice of the form $L\{1,1\}$.

Lemma. Let $A B C D$ be a convex quadrilateral with $\widehat{B}+\widehat{C} \leq 180^{\circ}$, $\widehat{C}+\widehat{D} \leq 180^{0}$ (Fig. 5a). Let $T$ be a point such that $\overrightarrow{B T}=\overrightarrow{A D}$ and let us call $(A B D)=E,(B T C)=E_{v},(D T C)=E_{h}$. Then $(D B C)=E+E_{v}+E_{h},(A B C)=E+E_{h},(A C D)=E+E_{v}$.

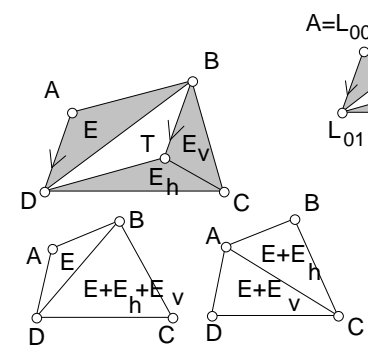

a

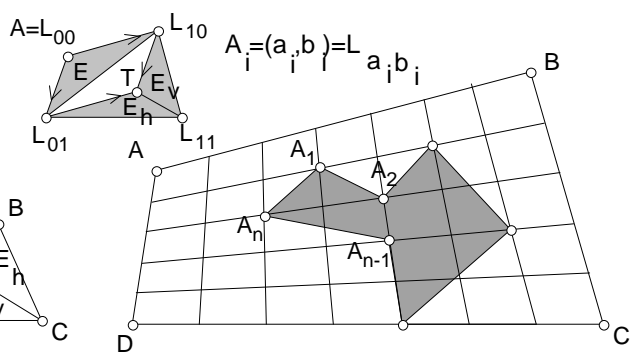

b

\section{Figure 5}

The following generalization of this lemma is our main result. The algebraic notation will be explained shortly after: 
Theorem 1. Let us consider a convex distorted lattice $L_{A B C D}\{m, n\}$ for which we assume without loss of generality that for its interior angles hold $\widehat{B}+\widehat{C} \leq 180^{\circ}, \widehat{C}+\widehat{D} \leq 180^{\circ}$ (Fig. 5b). Let $T$ be a point such that $\overrightarrow{L_{10} T}=\overrightarrow{L_{00} L_{01}}$ and call $\left(L_{01} L_{00} L_{10}\right)=E$, $\left(L_{10} T L_{11}\right)=E_{v},\left(L_{01} T L_{11}\right)=E_{h}$. If $A_{1} A_{2} \ldots A_{k}$ is a simple lattice polygon and $A_{i}=L_{a_{i} b_{i}}$ then

$$
\begin{aligned}
& \pm\left(A_{1} A_{2} \ldots A_{k}\right)=\left|\begin{array}{cccccccccc}
1 & 1 & 1 & 1 & \cdots & 1 & 1 & \\
& a_{1} & a_{2} & a_{3} & \cdots & a_{k-2} & a_{k-1} & a_{k} & a_{1} \\
& b_{1} & b_{2} & b_{3} & \cdots & b_{k-2} & b_{k-1} & b_{k} & b_{1} \\
1 & 1 & 1 & 1 & \cdots & 1 & 1 & &
\end{array}\right| E \\
& +\left|\begin{array}{ccccccccc}
a_{2} & a_{3} & a_{4} & a_{5} & \cdots & a_{k-4} & a_{k-3} & \\
& a_{1} & a_{2} & a_{3} & \cdots & a_{k-2} & a_{k-1} & a_{k} & a_{1} \\
& b_{1} & b_{2} & b_{3} & \cdots & b_{k-2} & b_{k-1} & b_{k} & b_{1} \\
a_{1} & a_{2} & a_{3} & a_{4} & \cdots & a_{k-1} & a_{k} & &
\end{array}\right| E_{h} \\
& +\left|\begin{array}{lllllllll}
b_{1} & b_{2} & b_{3} & b_{4} & \cdots & b_{k-1} & b_{k} & & \\
& a_{1} & a_{2} & a_{3} & \cdots & a_{k-2} & a_{k-1} & a_{k} & a_{1} \\
& b_{1} & b_{2} & b_{3} & \cdots & b_{k-2} & b_{k-1} & b_{k} & b_{1} \\
b_{2} & b_{3} & b_{4} & b_{5} & \cdots & b_{k} & b_{1} & &
\end{array}\right| E_{v}
\end{aligned}
$$

The + sign appears exactly when the orientation $\left(A_{1}, A_{2}, \ldots, A_{k}\right)$ of $A_{1} A_{2} \ldots A_{k}$ is its positive orientation in the plane. The assumption about the angles of $A B C D$ effects on the actual result but obviously it is not crucial. It basically organizes one's thought towards nicely arranged convex quadrilaterals. $E_{h}, E_{v}$ are a measure of how far is $L_{A B C D}\{m, n\}$ from being regular.

In the limit case $\widehat{D}+\widehat{C}=180^{\circ}, \widehat{B}+\widehat{C}=180^{\circ}$, i.e. whenever $A B C D$ is a parallelogram or equivalently whenever $L_{A B C D}\{m, n\}$ is the restriction of a usual infinite lattice in an appropriate finite piece of it, we get the

Corollary. Let us consider a convex distorted lattice $L_{A B C D}\{m, n\}$ for which $A B C D$ is a parallelogram (i.e. our lattice is a finite restriction of an infinite regular lattice), and let us call $\left(L_{01} L_{00} L_{10}\right)=E$. If $A_{1} A_{2} \ldots A_{k}$ is a simple lattice polygon and $A_{i}=\left(a_{i}, b_{i}\right)$ then

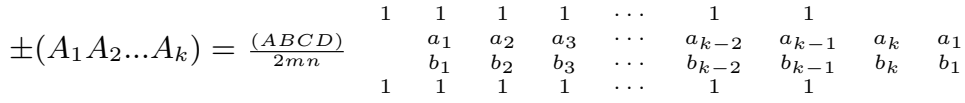

If moreover $L_{A B C D}\{m, n\}$ is part of the regular lattice of the integers in the plane, or more generally whenever the lattice quadrilaterals have unit area, the last formula becomes 


$$
\pm\left(A_{1} A_{2} \ldots A_{k}\right)=\frac{1}{2}\left|\begin{array}{ccccccccc}
1 & 1 & 1 & 1 & \cdots & 1 & 1 & & \\
& a_{1} & a_{2} & a_{3} & \cdots & a_{k-2} & a_{k-1} & a_{k} & a_{1} \\
& b_{1} & b_{2} & b_{3} & \cdots & b_{k-2} & b_{k-1} & b_{k} & b_{1} \\
1 & 1 & 1 & 1 & \cdots & 1 & 1 & &
\end{array}\right| .
$$

The algebraic notation used above is explained by the

Definition 2. A long determinant

$$
A=\left|\begin{array}{llllllll}
c_{1} & c_{2} & c_{3} & c_{4} & \cdots & c_{r-1} & & \\
& a_{1} & a_{2} & a_{3} & \cdots & a_{r-2} & a_{r-1} & a_{r} \\
& b_{1} & b_{2} & b_{3} & \cdots & b_{r-2} & b_{r-1} & b_{r} \\
d_{1} & d_{2} & d_{3} & d_{4} & \cdots & d_{r-1} & &
\end{array}\right|
$$

is an orthogonal array of numbers in 4 rows and $r+1 \geqq 3$ columns. The last two places of each of the first and the last row are empty, as are the second and third places of the first column. This symbol is defined as another shorthand of

$$
\begin{aligned}
\sum_{1}^{r-1} c_{i} a_{i} b_{i+1}-\sum_{1}^{r-1} d_{i} b_{i} a_{i+1}=c_{1} a_{1} b_{2}+c_{2} a_{2} b_{3}+\cdots+ \\
c_{r-1} a_{r-1} b_{r}-d_{1} b_{1} a_{2}-d_{2} b_{2} a_{3}-\cdots-d_{r-1} b_{r-1} a_{r} .
\end{aligned}
$$

Long determinants can have an arbitrary long row size. In many ways they resemble the usual determinants and they can actually be defined in terms of them. For example check out that the long determinant

$$
\left|\begin{array}{ccccc}
1 & 1 & 1 & & \\
& a_{1} & a_{2} & a_{3} & a_{1} \\
& b_{1} & b_{2} & b_{3} & b_{1} \\
1 & 1 & 1 & &
\end{array}\right|
$$

equals the usual determinant

$$
\left|\begin{array}{ccc}
a_{1} & a_{2} & a_{3} \\
b_{1} & b_{2} & b_{3} \\
1 & 1 & 1
\end{array}\right| .
$$

Thus we believe that the name we assigned to them is a suitable one.

Although long determinants posses many interesting properties we shall not be interested in them here. We are mainly going to use them as a concise symbol neatly expressing our results. 


\section{Technicalities}

We now develop the necessary technical details for the proofs of the results stated in Section 1. These details consist of elementary results in Euclidean Geometry and Algebra. Accordingly, our treatment will be elementary. The reader can skip most of this section, hopefully without conceptual difficulties in following Sections 3 and 4. One can even skip the whole section and return to it only when he/she finds it necessary.

We start by presenting a concise summary of well known facts about polygons which are important to us here. But beware, not all of their their proofs are trivial. The interested reader can try to prove some of them but it is possibly better to consult appropriate books ([1], [2] and many others). Of course it will be assumed tacitly that we work within one of the usual axiomatic definitions of Euclidean Geometry (for example cf. [2]).

Next we prove in the form of small lemmas some not really deep properties of polygons, but of a special importance to our goals in this article. The proofs to be presented are not unique or the best ones. They are included here just for the shake of rigorousness since most of them are not found in the literature. Our arguments will be purely geometric.

2.1. Known facts about polygons. First we need to clarify the notion of polygon in which we are interested here.

We care about simple closed polygons (or just polygons from now on) in the usual sense:

Combinatorial definition: A polygon is a collection of a finite sequence of distinct points $\left(A_{1}, A_{2}, \cdots, A_{n}\right)(n \in \mathbb{N}, n \geqq 3)$ in the plane (called the polygon's vertices) and of the line segments $\left(A_{1} A_{2}, A_{2} A_{3}, \cdots, A_{n-1} A_{n}, A_{n} A_{1}\right)$ (called the polygon's sides) such that: each vertex belongs to exactly two sides and every pair of distinct sides shares at most one point, in which case this point is a vertex. Finally, two consecutive sides (in a cyclic sense) are not allowed to lie on the same line. This polygon will be denoted as $\left\langle A_{1} A_{2} \cdots A_{n}\right\rangle$ and it will be called $n$-gon. We agree to consider any other polygon produced by a cyclic permutation of $\left(A_{1}, A_{2}, \cdots A_{n}\right)$ as coinciding with $<A_{1} A_{2} \cdots A_{n}>$. The set of cyclic permutations in which $\left(A_{1}, A_{2}, \cdots A_{n}\right)$ belongs will be called the orientation of the polygon. 
Point set definition: The point set $P$ of the plane consisting of the union of the polygon's sides can be obtained as union of the sides of another polygon, namely the one defined by the sequence $\left(A_{n}, A_{n-1} \cdots, A_{1}\right)$ and the corresponding sequence of sides $\left(A_{n} A_{n-1}, A_{n-1} A_{n-2}, \cdots, A_{2} A_{1}, A_{1} A_{n}\right)$ (check that this is indeed a polygon!). $P$ is called Euclidean Polygon, or just polygon if no confusion arises and either $\left(A_{1}, A_{2}, \cdots, A_{n}\right),\left(A_{n}, A_{n-1}, \cdots, A_{1}\right)$ define it. We write $P=A_{1} A_{2} \cdots A_{n}=A_{n}, A_{n-1}, \cdots, A_{1}$. We also say that $P$ has two orientations: $\left(A_{1}, A_{2}, \cdots, A_{n}\right)$ and $\left(A_{n}, A_{n-1}, \cdots, A_{1}\right)$. These are by definition all the orientations of $P$ and they are distinct. Exactly one of them will be called the positive orientation of $P$ in the plane:

Loosely speaking, the positive orientation of $P$ in the plane is the one for which a journey around the polygon according to this orientation results to a net full anti-clockwise twist in respect to a fixed interior point. Another way to express this idea is by saying that the positive orientation in the plane is the one for which during a journey around the polygon following this orientation, our left hand always points towards the interior of the polygon (here again for the sake of simplicity we have used a somehow loose mathematical terminology). Exactly one of the two orientations of $P$ is its positive orientation in the plane (Fig. 6a). We are going to follow the usual terminology

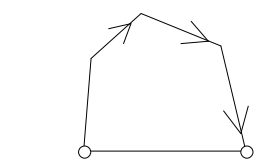

Positive orientation

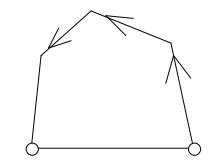

Negative orientation

a
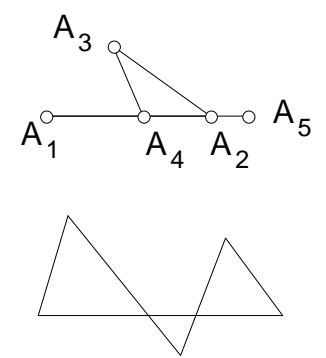

Not polygons

b

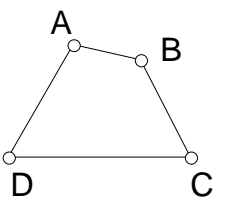

A

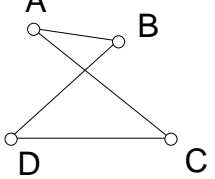

C

Figure 6

calling polygons with 3,4 , etc. sides as triangles, quadrilaterals etc., 
and we will be using the terms parallelogram, square and so on, in the usual sense.

The above definition of a polygon does not allow two distinct sides to share a common nontrivial sub-segment of each, or to share even just one interior point of each (Fig. $6 \mathrm{~b}$ ). Note also that $A B C D$ is not the same as $A B D C$ (Fig. 6c). So the order of the vertices (i.e., the sequence in the definition) is important!

Very often one needs to consider as polygons some limit cases of polygons (as defined above). For example it is often convenient to drop in the definition the condition that two consecutive sides are not allowed to lie on the same line. Then we talk about n-gons in the broad sense. In Figure 7a, $P=A C B D$ is a polygon of 4 -sides in a broad sense. Actually it is a virtual 3 -gon (triangle) coinciding with $A D C$.

Observe that if $B C$ is a line and $A, D$ are points in different halfplanes with respect to the line $B C$ then $A B D C$ is always a quadrilateral or at least a quadrilateral in the broad sense (Fig. 7a,b). In

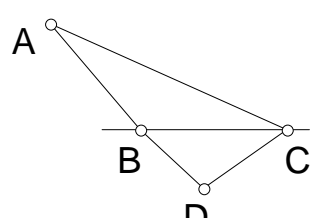

a

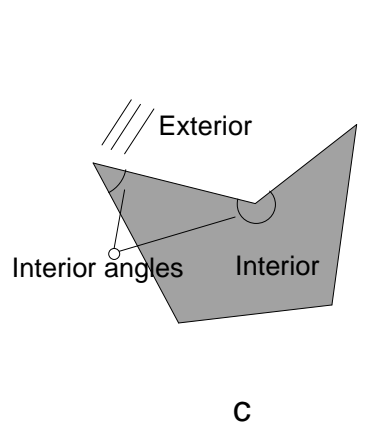

C

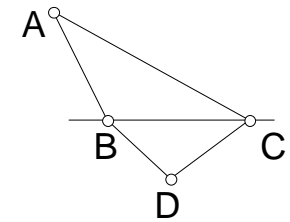

b

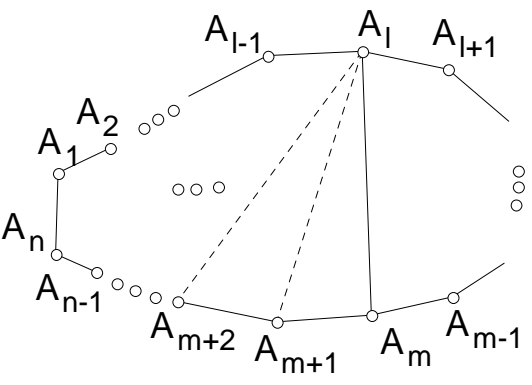

d

FiguRe 7

what follows, most lemmas and propositions hold for polygons in the broad sense as well. 
The following properties of the polygons are known to be true:

- Any polygon divides the plane into two disjoint regions and is the boundary of each (Fig. 7c).

- Any two points $K, L$ of either of the above two regions, can be connected by a finite sequence of segments $K A_{1}, A_{1} A_{2}, \cdots, A_{n-1} A_{n}$, $A_{n} L$ so that all segments lie entirely in this region.

- Any line segment with an end point in one of the regions and the other endpoint in the other region intersects the polygon at least in one point.

- Exactly one of the regions is bounded, i.e., the distance of any two of its points is less than a constant real number.

The bounded region is called the interior of the polygon and the other (unbounded) region is called the exterior of the polygon. Note that the points of the polygon itself do not belong to either of these two regions. The union of a polygon with its interior will be called a polygonal region; the vertices, the sides, and the orientation of the polygon will be called vertices, sides, interior and orientation of the polygonal region as well. The polygon itself will be called corresponding polygon to the polygonal region.

If $A_{1} A_{2} \cdots A_{n}$ is a polygon, its polygonal region will be denoted as $\overline{A_{1} A_{2} \cdots A_{n}}$.

Some other equally notable results require the notion of convex polygons:

According to one of many equivalent definitions, a polygon is convex if the line defined by any one of its sides, leaves all other sides of the polygon at the same half-plane with respect to this line.

An equivalent definition states that the polygon is convex if all interior angles of the polygon are convex (i.e., less than $180^{\circ}$ ). For a polygon $A_{1} A_{2} \cdots A_{n}$, an angle $A_{i-1} \widehat{A}_{i} A_{i+1}($ indices $\bmod n)$ is called interior, if the points of the angle not on the sides and close enough to the vertex, all lie in the interior of the polygon. We will be denoting the interior angle $A_{i-1} \widehat{A}_{i} A_{i+1}$ simply by $\widehat{A}_{i}$ (Fig. 7c). All angles here are defined as usual without reference to any extra equipment like orientation etc.

Yet another definition of a convex polygon is the one asking that any line segment with endpoints on the corresponding polygon region, lies itself on this region. 
For a quadrilateral, there exists an even funnier definition (or else property) for convexity: $P=<A B C D>$ is convex iff the diagonals $A C, B D$ intersect in an interior point of each.

For convex polygons the following properties are known to be true: - Any line segment with one end point in the interior and the other in exterior of a convex polygon intersects the polygon exactly once. - A line with points in both the interior and the exterior of a convex polygon intersects the polygon in exactly two points.

- A line segment with endpoints on a convex polygon lies except from its endpoints entirely in the interior of the polygon. Returning to the properties of arbitrary polygons (not necessarily convex) we should mention that there is defined a function measuring the size of any polygonal region, called area of the polygon. There exist many ways to define the important notion of area. Since the present article refers to elementary Euclidean Geometry we follow the usual elementary way of defining area following the rules:

- To each polygonal region is assigned an area which is a non negative number.

- Equal polygonal regions have equal areas.

- A square with side of unit length has unit area.

- A polygonal region partitioned into a finite number of other polygonal regions, has area equal to the sum of the areas of the polygonal regions in its partition (addition property).

For the shake of rigorousness we should mention that two polygonal regions in the plane are said equal if one can assume the position of the other in the plane after a sequence of finitely many Euclidean moves in the space.

Also, a finite partition of a polygonal region means that there exist finitely many polygonal regions so that

- any two of them do not share a common interior point and

- the union of these regions (as a subset of the plane) is our original polygonal region.

The trick to make actual calculations with areas is given by the triangulation property of the polygonal regions:

Any polygonal region can be partitioned into triangular regions, and moreover this can be done by drawing internal diagonals of the polygonal region.

Diagonal means a line segment with endpoints two non consecutive vertices of the polygonal region, and internal diagonal means that the whole diagonal lies in the polygonal region. 
So then in order to calculate the area of a polygonal region, one needs to provide a formula for the area of an arbitrary triangular region, to triangulate the given polygonal region and finally to use the addition property of the area.

A couple of remarks on internal diagonals are in order here:

An internal diagonal $A_{i} A_{j}(1 \leq l<m \leq n-2)$ of a polygonal region $\bar{P}=\overline{A_{1} A_{2} \cdots A_{n}}$ partitions the region into two other polygonal regions: $\bar{P}_{1}=\overline{A_{1} A_{2} \cdots A_{l} A_{m} A_{m+1} \cdots A_{n}}, \overline{P_{2}}=\overline{A_{l} A_{l+1} \cdots A_{m-1} A}$ (Fig. 7d).

An internal diagonal of any of these two regions is an internal diagonal of $\overline{A_{1} A_{2} \cdots A_{n}}$ as well. The only common part of $\bar{P}_{1}, \overline{P_{2}}$ is their common side $A_{l} A_{m}$. The orientations $\left(A_{1}, A_{2}, \cdots, A_{l}, A_{m}, A_{m+1}, \cdots\right.$ $\left.A_{n}\right),\left(A_{l}, A_{m+1}, \cdots, A_{l-1}, A_{l}\right)$ of $\bar{P}_{1}, \bar{P}_{2}$ are called induced by the orientation $\left(A_{1}, A_{2}, \cdots, A_{n}\right)$ of $\bar{P} ; \bar{P}, \bar{P}_{1}, \bar{P}_{2}$ equipped with these orientations are called similarly oriented. Similarly for the other orientations of these polygonal regions. Whenever $\bar{P}$ is equipped with its positive orientations in the plane, then the induced orientations on $\bar{P}_{1}, \bar{P}_{2}$ are their positive orientations in the plane as well.

Now especially for the polygonal region of a quadrilateral, it is true that both its diagonals or exactly one is internal depending on if the quadrilateral is convex or not. By the way note that the existence of at least one internal diagonal implies immediately that the sum of the interior angles of any quadrilateral equals $360^{\circ}$. In the case of a convex $A B C D$ each diagonal, being an internal diagonal, partitions $\overline{A B C D}$ into two triangular regions. Note that for a convex $A B C D$ again, the line $B D$ leaves the vertices $A, C$ in different half-planes, and similarly for the line $A C$.

Let us close mentioning that if $A, B, C, D$ are points in the plane such that $\overrightarrow{A B}=\overrightarrow{D C}$ then $A B C D$ is a parallelogram. All parallelograms are convex polygons.

2.2. Some more facts about polygons and a property of long determinants. The reader should be aware of the fact that although most of the results in the following lemmas are intuitively clear, their proofs can be tiresome. One should occasionally recall the previous remarks on polygons. Many arguments can be considerably shortened if one assumes a few more facts about polygons as given.

Our first lemma provides us with a tool used for an induction argument in the proof of our main result. 
The exposition will be considerably facilitated if for a polygonal region $\overline{A_{1} A_{2} \cdots A_{n}}$ we call a pair of vertices of the form $A_{r}, A_{r+2}$ (indices $\bmod n$ ) as "next to side" vertices.

Lemma 1. For any polygonal region with $n$ vertices $(n \geqq 4)$ there exists an internal diagonal joining a next to side pair of vertices.

In other words, there exists an internal diagonal which partitions our polygonal region in at least one triangular region.

Proof. We prove it by a total induction argument:

For $n=4$, clearly any internal diagonal has the required property.

Let us suppose that the proposition is true for all values of $n$ up to some value $k \geqq 4$.

We are going to prove that the proposition is also true for $n=k+1$ and thus we will be done.

So let $\bar{P}=\overline{A_{1} A_{2} \cdots A_{k+1}}$ be a polygonal region. We will prove the existence of a diagonal with the desired property by a descending induction argument:

Let $A_{l} A_{m}, 1 \leqq l<m \leqq n-2$ be an internal diagonal of $\bar{P}$ (Fig. 7d) and let us call $\bar{P}_{1}=\overline{A_{1} A_{2} \cdots A_{l} A_{m} A_{m+1} \cdots A_{n}}$. Let us also call $N\left(\bar{P}_{1}\right)$ the number of vertices of $\bar{P}_{1}$.

If we were lucky enough to have $N\left(\bar{P}_{1}\right)=3$, then of course this would translate to $d_{1}$ being an internal diagonal of $\bar{P}$ with the desired property.

So from now on let us assume that $N\left(\bar{P}_{1}\right) \geqq 4$.

Since $4 \leq N\left(\bar{P}_{1}\right) \leq(k+1)-1=k$, the induction hypothesis provides an internal diagonal $d_{1}$ of $\bar{P}_{1}$ joining a next to side pair of vertices of $\bar{P}_{1}$, say $\left\{T_{1}, T_{1}^{\prime}\right\} . d_{1}$ is an internal diagonal of $\bar{P}$ as well. If $\left\{T_{1}, T_{1}^{\prime}\right\} \neq\left\{A_{l}, A_{m+1}\right\}$ and $\left\{T_{1}, T_{1}^{\prime}\right\} \neq\left\{A_{l-1} A_{m}\right\}$ then $\left\{T_{1}, T_{1}^{\prime}\right\}$ is a next to side pair of vertices of $\bar{P}$ as well, making the internal diagonal $d_{1}$ of $\bar{P}_{1}$ one of the desired.

On the other hand, if not both $\left\{T_{1}, T_{1}^{\prime}\right\} \neq\left\{A_{l}, A_{m+1}\right\},\left\{T_{1}, T_{1}^{\prime}\right\} \neq$ $\left\{A_{l-1} A_{m}\right\}$ are true, let us assume without any loss of generality that $\left\{T_{1}, T_{1}^{\prime}\right\}=\left\{A_{l}, A_{m+1}\right\}$. Then:

Let $N\left(\bar{P}_{2}\right)$ be the number of vertices of the polygonal region $\bar{P}_{2}=\overline{A_{1} A_{2} \cdots A_{l} A_{m+1} A_{m+2} \cdots A_{n}}$.

If $N\left(\bar{P}_{2}\right)=3$, this would translate to $T_{1} T_{1}^{\prime}=A_{l} A_{m+1}$ being an internal diagonal of $\bar{P}$ with the desired property.

So from now on let us assume that $N\left(\bar{P}_{1}\right) \geqq 4$. 
Then since $4 \leq N\left(\bar{P}_{2}\right)=N\left(\bar{P}_{1}\right)-1 \leq k$ the induction hypothesis provides to us an internal diagonal $d_{2}$ of $\bar{P}_{2}$ joining a next to side pair of vertices of $\bar{P}_{2}$, say $\left\{T_{2}, T_{2}^{\prime}\right\} . d_{2}$ is an internal diagonal of $\bar{P}$ as well.

With a similar argument as for $d_{1}$ we show that either $d_{2}$ is an internal diagonal of $\bar{P}$ with the desired property, or else without loss of generality $\left\{T_{2}, T_{2}^{\prime}\right\}=\left\{A_{l}, A_{m+2}\right\}$.

We continue in this way and we

(I) either find an internal diagonal of $\bar{P}$ with the desired property for $\bar{P}$, or else

(II) we form a sequence of internal diagonals $d_{1}, d_{2}, \cdots$ of $\bar{P}$ such that none has the desired property of $\bar{P}$ but which have the property: each $d_{i}$ is a diagonal of a polygonal region $\bar{P}_{i}$ with number of vertices $N\left(\bar{P}_{i}\right)$ such that $4 \leq N\left(\bar{P}_{1}\right)=k$ and $4 \leq N\left(\bar{P}_{i}\right)=N\left(\bar{P}_{i-1}\right)-1$ for $i \geqq 2$.

Clearly (II) leads to a contradiction as follows: Since the number $N\left(\bar{P}_{i}\right)$ of vertices of $\bar{P}_{i}$ decreases by 1 as $i$ increases by 1 , inevitably $N\left(\bar{P}_{i}\right)$ would have to be less than 4 for some $i$, which is a contradiction because of $4 \leq N\left(\bar{P}_{i}\right)$ which is also supposed to be true.

Thus (I) holds and we have finished.

The next lemma is an almost obvious fact and any proof of it depends on how much one accepts as already proved to be true.

Lemma 2. A line segment joining two interior points in opposite sides of a convex quadrilateral partitions it into two convex quadrilaterals.

Proof. Let $K, L$ be points on the sides $A B, C D$ respectively of the convex quadrilateral $A B C D$. We will prove the result for $A K L D$; similar arguments will prove it for $K B C L$ as well.

It is enough to show that every side of $A K L D$ defines a line that leaves all other sides at the same half plane (with respect to the line).

Since $A B C D$ is convex, the line $A B$, i.e. the line $A K$, leaves at the same half-plane the whole $\overline{A B C D}$. Again because $A B C D$ is convex, the segment $L K$ lies in $\overline{A B C D}$.

Thus the line $A K$ leaves at the same half-plane all three segments $A D, D L, K L$.

Similarly the line $D L$ leaves at the same half-plane all three segments $A D, A K, K L$, and line $A D$ leaves at the same half plane all three segments $A K, K L, D L$. 
Finally showing that the points $A, D$ lie at the same half-plane with respect to the line $K L$ would imply that line $K L$ leaves at the same half-plane the segments $A K, A D, D L$; and paired with the above facts about lines $A K, D L, A D$, we would have finished.

Note that since $A B C D$ is convex, the interior points of the segment $K L$ lie in the interior of $A B C D$, the points of the line $K L$ exterior to the segment $K L$ lie in the exterior of $A B C D$, and the points $K, L$ lie on $A B C D$.

Now if we assume for a moment that the points $A, D$ lie at different half planes with respect to the line $K L$, the segment $A D$ would have to intersect the line $K L$, say at a point $S$. The point $S$ lying at segment $A B$, does not lie at the exterior of $A B C D$ and so it has to be a point of the segment $K L$ (Fig. 8a).

$S$ cannot coincide with an end point of $K L$. If for example it coincides with $K$, then $K$ as a point lying on both $A B, A D$ should be their unique common point $A$, a contradiction by assumption.

$S$ cannot either be an interior point of $K L$, since otherwise it would have to lie at the interior of $A B C D$, a contradiction because $S$ as a point of the segment $A B$ lies on $A B C D$.

Thus the points $A, D$ lie indeed at the same half-plane with respect to the line $K L$ as wanted.

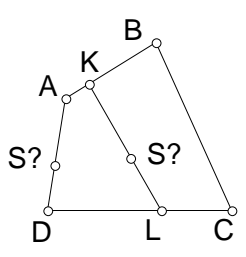

a

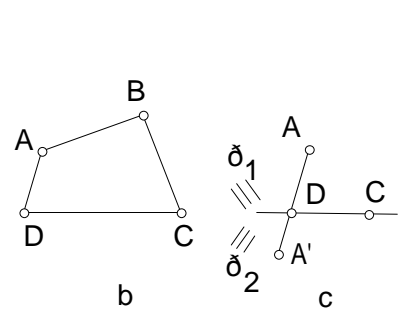

FiguRE 8

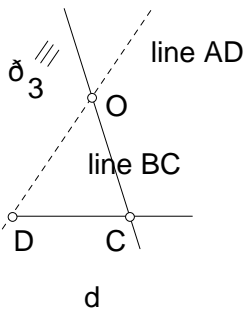
sense, i.e., no three of its vertices are collinear, then $A K L D$ is also a quadrilateral in the usual sense. For if for example $D, A, K$ are collinear then the segments $D A, A K$ lie on the same line. Since the segments $A K, A B$ lie at the same line, we get that the segments $D A, A B$ lie at the same line, which gives that the points $D, A, B$ are collinear, a contradiction. 
We now give a property about the angles of a quadrilateral.

Lemma 3. (a) For any quadrilateral there exist three consecutive interior angles $\widehat{X}, \widehat{Y}, \widehat{Z}$ such that $\widehat{X}+\widehat{Y} \leq 180^{\circ}$ and $\widehat{Y}+\widehat{Z} \leq 180^{\circ}$. (b) If $A B C D$ is a convex quadrilateral with interior angles such that $\widehat{B}+\widehat{C} \leq 180^{\circ}, \widehat{C}+\widehat{D} \leq 180^{\circ}$ then the half-lines $D A, C B$ intersect (or they are parallel and lie) at the same half-plane with respect to the line $C D$ at which the line segment $A B$ lies. Moreover if $O$ is the intersection point of the half-lines $D A, C B$ then $A, B$ lie at the interior of the segments DO,CO respectively. Similar facts hold for the half-lines $B A, C D$.

Proof. (a) Let $\widehat{A}, \widehat{B}, \widehat{C}, \widehat{D}$ be the interior angles of $A B C D$ with vertices at $A, B, C, D$ respectively.

The relation $\widehat{A}+\widehat{B}+\widehat{C}+\widehat{D}=360^{\circ}$ implies that either $\widehat{B}+\widehat{C} \leq 180^{\circ}$ or $\widehat{A}+\widehat{D} \leq 180^{\circ}$.

Without loss of generality let us assume that $\widehat{B}+\widehat{C} \leq 180^{\circ}$.

Then again the relation $\widehat{A}+\widehat{B}+\widehat{C}+\widehat{D}=360^{\circ}$ implies that either $\widehat{C}+\widehat{D} \leq 180^{\circ}$ or $\widehat{A}+\widehat{B} \leq 180^{\circ}$.

Without loss of generality let us assume that $\widehat{C}+\widehat{D} \leq 180^{\circ}$. Then $\widehat{B}, \widehat{C}, \widehat{D}$ have the required property.

(b) Let $\pi_{1}, \pi_{2}$ be the two half-planes with respect to the line $C D$. Since $A B C D$ is convex, both $A, B$ lie on just one of $\pi_{1}, \pi_{2}$ say on $\pi_{1}$.

It is certainly true that the half-line $D A$ lies entirely in $\pi_{1}$ (Fig. 8b); for if not, then by assuming $A^{\prime}$ to be a point of the half-line $D A$ in $\pi_{2}$ then the line $C D$ intersects the segment $A A^{\prime}$ in an interior point. Of course, this intersection point is necessarily the point $D$ (Fig. 8c). But $D$ as the origin of the half-line $D A$ cannot lie in the interior of any segment $A A^{\prime}$ of this half-line and we arrived in a contradiction.

Similarly it is true that the half-line $C B$ lies entirely in $\pi_{1}$.

So we proved that the half-lines $D A, C B$ lie at the same half-plane $\pi_{1}$ with respect to the line $C D$.

Now the relation $\widehat{C}+\widehat{D} \leq 180^{\circ}$ i.e. $B \widehat{C} D+A \widehat{D} C \leq 180^{\circ}$ is well known to imply that the half-lines $D A, C B$ are parallel or intersect; note that generally there exist three relative positioning cases for two half-lines: they intersect, they are parallel or they do not intersect without being parallel.

Of course if they intersect say at $O$, this point ought to lie on $\pi_{1}$ as well (Fig. 8d). In this case, since $A B C D$ is convex, both $A, D$ 
lie at the same half-plane, call it $\pi_{3}$, with respect to the line $B C$ i.e. with respect to the line $O C$. (Note that the points $O, C$ indeed define a line, i.e. $C$ does not coincide with $O$ for otherwise the line $D O$ i.e. $D A$ would coincide with line $D C$, a contradiction).

The part of the line $D O$ which lies at $\pi_{3}$ is the half-line $O D$. Thus $A$ has to lie on the half-line $O D$. Since $A$ also lies at the half-line $D O$, we conclude that it lies on the intersection of the half-lines $O D$ and $D O$ which is the segment $O D$. Of course $A$ cannot coincide with any of $D, O$ since otherwise $A B C D$ would not satisfy the defining conditions for a polygon. So $A$ is an interior point of the segment $D O$. Similarly we show that $B$ lies in the interior of the segment $C O$.

The next lemma investigates the way by which the property of "nice arranged position" is inherited to the two quadrilaterals at which a convex quadrilateral is partitioned by a line segment joining two opposite sides.

Lemma 4. Let $A B C D$ be a convex quadrilateral with interior angles such that $\widehat{B}+\widehat{C} \leq 180^{\circ}, \widehat{C}+\widehat{D} \leq 180^{\circ}$ and $K, N, L, M$ be interior points of the sides $A B, B C, C D, D A$ respectively such that $\frac{A K}{K B}=$ $\frac{D L}{L C}$ and $\frac{B N}{N C}=\frac{A M}{M D}$. Then in the quadrilaterals $A K L D, K B C D$, $A B N M, N M C D$ the following hold respectively about their interior angles: (a) $\left(\widehat{K}+\widehat{L} \leq 180^{\circ}, \widehat{D}+\widehat{L} \leq 180^{\circ}\right)$, (b) $\left(\widehat{L}+\widehat{C} \leq 180^{\circ}, \widehat{B}+\right.$ $\left.\widehat{C} \leq 180^{\circ}\right)$, (c) $\left(\widehat{B}+\widehat{N} \leq 180^{\circ}, \widehat{M}+\widehat{N} \leq 180^{\circ}\right)$, (d) $(\widehat{N}+\widehat{C} \leq$ $\left.180^{\circ}, \widehat{D}+\widehat{C} \leq 180^{\circ}\right)$

Proof. We are only going to prove (a) and (b) since the rest are proved in a similar way. Actually in a sense, (c) and (d) are just a repetition of (a) and (b). In order to keep a better track of the angles involved, we are going to use their usual three letter notation.

(a) Proof of $A \widehat{K} L+K \widehat{L} D \leq 180^{\circ}$ :

If $A B \| D C$ then we immediately have $A \widehat{K} L+K \widehat{L} D=180^{\circ}$.

If $A B \nVdash D C$, then the lines $A B, D C$ intersect, say at $O$ (Fig. 9a). By the previous lemma, $A$ lies on the segment $O B$. Since $K$ is a point of the segment $A B$, the point $A$ will also lie in the segment $O K$. This is equivalent to $O$ lying in the half-line $K A$. Similarly $O$ lies on the half-line $L D$.

So $O$ is the intersection point of the half-lines $K A, L D$. This is equivalent to $A \widehat{K} L+D \widehat{L} K<180^{\circ}$ i.e. $A \widehat{K} L+K \widehat{L} D<180^{\circ}$ as wanted. 
Proof of $A \widehat{D} L+D \widehat{L} K \leq 180^{\circ}$ :

Let $l_{1}, l_{2}$ be the parallel lines to the line $A D$ from $L, C$ respectively. Let also $L^{\prime}, C^{\prime}$ be the intersection points of the line $A B$ with $l_{1}, l_{2}$ respectively (Fig. 9b,c). Since $L$ is a point of the segment $D C$ it follows that $l_{1}, l_{2}$ lie at the same half-plane -say $(\pi)$ - with respect to the line $A D$ and $L^{\prime}$ is a point of the segment $A C^{\prime}$ such that $\frac{A L^{\prime}}{L^{\prime} C^{\prime}}$ $=\frac{D L}{L C}$.

Since $A B C D$ is convex, it totally lies at the same half plane with respect to the line $A D$. But the point $C$ of $A B C D$ lie with respect to the line $A D$ at the half-plane $(\pi)$. Thus $A B C D$ lies totally at $(\pi)$, and then the points $A, K, B, L^{\prime}, C^{\prime}$ lie on the same half-line $A x=(\pi) \cap A D$ of the line $A D$.

Then since $\frac{A L^{\prime}}{L^{\prime} C^{\prime}}=\left(\frac{D L}{L C}=\right) \frac{A K}{K B}$ we either have that

$K$ lies in the segment $A L^{\prime}$ and $B$ lies in the segment $A C^{\prime}$ (Fig. 9b)

or

$K$ lies in $L^{\prime} x-\left\{L^{\prime}\right\}$ and $B$ lies in $C^{\prime} x-\left\{C^{\prime}\right\}$ (Fig. 9c).

In the latter case we would have $A \widehat{D} C+B \widehat{C} D>A \widehat{D} C+D \widehat{C} C^{\prime}>$ $180^{\circ}$, a contradiction.

So the former case is true, which implies that $A \widehat{D} L+D \widehat{L} K \leq$ $A \widehat{D} L+D \widehat{L} L^{\prime}=180^{\circ}$.

(b) The relation $K \widehat{B} C+B \widehat{C} L \leq 180^{\circ}$ is none other than the relation $A \widehat{B} C+B \widehat{C} D \leq 180^{\circ}$ which holds by assumption.

The relation $K \widehat{L} C+L \widehat{C} B \leq 180^{\circ}$ can be proved in a similar manner as was proved relation $A \widehat{D} L+D \widehat{L} K \leq 180^{\circ}$ above, only now one has to draw the parallels from $D$ and $L$ to the line $B C$.

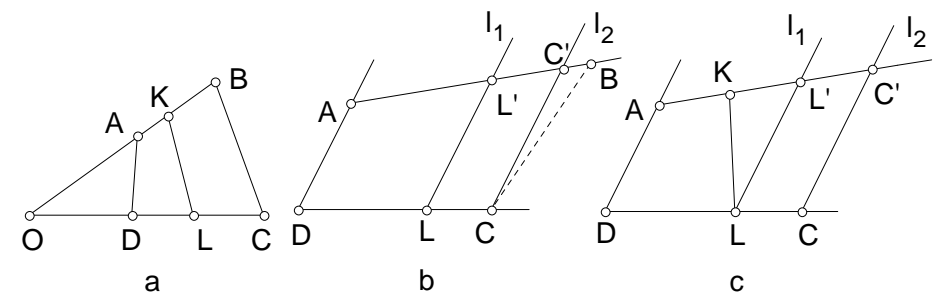

FiguRE 9

An immediate corollary concerning distorted lattices is the following. 
Lemma 5. In a convex distorted lattice $L_{A B C D}\{m, n\}$ all quadrilaterals $\overline{L_{k l} L_{t l} L_{t s} L_{k s}}, k, t \in\{0,1, \cdots, m\}, l, s \in\{0,1, \cdots, n\}, k<t$, $l<s$ with sides on the lattice segments are convex and for their interior angles hold $\widehat{L}_{t l}+\widehat{L}_{t s} \leq 180^{\circ}, \widehat{L}_{t s}+\widehat{L}_{k s} \leq 180^{\circ}$.

Proof. By Lemma 2 the segment $L_{k 0} L_{k n}$ partitions $\overline{A B C D}$ into two convex quadrilateral regions $\overline{A L_{k 0} L_{k n} D}, \overline{L_{k 0} B C L_{k n}}$ (Fig. 10a). Lemma 4 assures us that for the interior angles of $\overline{L_{k 0} B C L_{k n}}$ hold: $\widehat{B}+\widehat{C} \leq 180^{\circ}, \widehat{C}+\widehat{L}_{k n} \leq 180^{\circ}$.

By Lemma 2 the segment $L_{t 0} L_{t n}$ partitions the region $\overline{L_{k 0} D L_{k n} C}$ into two convex quadrilateral regions $\overline{L_{k 0} L_{t 0} L_{t n} L_{k n}}, \overline{L_{t 0} B C L_{t n}}$.

Lemma 4 assures us that for the interior angles of $\overline{L_{k 0} L_{t 0} L_{t n} L_{k n}}$ hold: $\widehat{L}_{t 0}+\widehat{L}_{t n} \leq 180^{\circ}, \widehat{L}_{t n}+\widehat{L}_{k n} \leq 180^{0}$.

By Lemma 2 the segment $L_{k l} L_{t l}$ partitions $\overline{L_{k 0} L_{t 0} L_{t n} L_{k n}}$ into two convex quadrilateral regions $\overline{L_{k 0} L_{t 0} L_{t l} L_{k l}}, \overline{L_{k l} L_{t l} L_{t n} L_{k n}}$.

Lemma 4 assures us that for the interior angles of $\overline{L_{k l} L_{t l} L_{t n} L_{k n}}$ hold: $\widehat{L}_{t l}+\widehat{L}_{t n} \leq 180^{\circ}, \widehat{L}_{t n}+\widehat{L}_{k n} \leq 180^{\circ}$.

Finally by Lemma 2 the segment $L_{k s} L_{t s}$ partitions $\overline{L_{k l} L_{t l} L_{t n} L_{k n}}$ into two convex quadrilateral regions $\overline{L_{k l} L_{t l} L_{t s} L_{k s}}, \overline{L_{k s} L_{t s} L_{t n} L_{k n}}$.

Lemma 4 assures us that for the interior angles of $\overline{L_{k l} L_{t l} L_{t s} L_{k s}}$ hold: $\widehat{L}_{t l}+\widehat{L}_{t s} \leq 180^{0}, \widehat{L}_{t s}+\widehat{L}_{k s} \leq 180^{0}$.
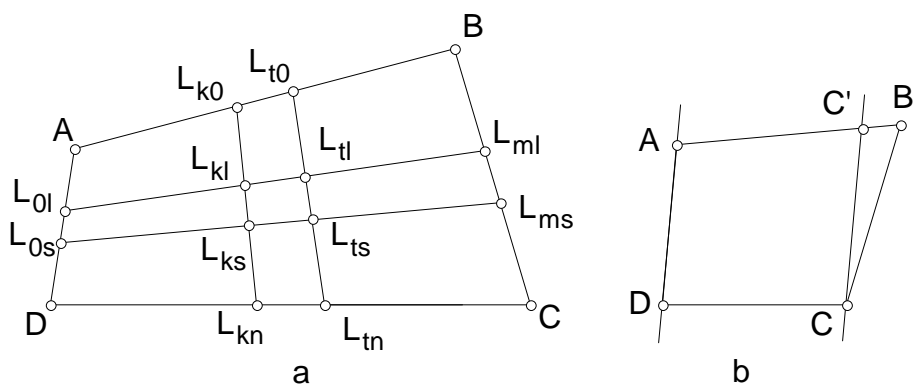

FigURE 10

The following lemma deals with some extremely useful for our purposes point positioning properties.

Lemma 6. Let $A B C D$ be a convex quadrilateral with interior angles such that $\widehat{B}+\widehat{C} \leq 180^{\circ}, \widehat{C}+\widehat{D} \leq 180^{\circ}$ and let $T, M, N$ be points such 
that $\overrightarrow{B T}=\overrightarrow{M C}=\overrightarrow{A D}$. Then (a) $T$ is interior point of $A B C D$ (or lies on the segment $A B$ whenever $A B \| C D$ ). Even more is true: $T$ is interior point of $D B C$ whenever $A B \nVdash C D$, (b) $M$ is exterior point of $A B C D$, or lies on the segment $B C$ whenever $A D \| B C$, (c) $A B M C$ is a convex quadrilateral, or just a triangle whenever $A B \| C D$ or $A D \| B C$.

Note that $T$ is also defined by the relation $\overrightarrow{D T}=\overrightarrow{A B}$. Defining the point $M^{\prime}$ by the relation $\overrightarrow{M^{\prime} C}=\overrightarrow{A B}$ similar facts to the above hold for the points $T, M^{\prime}$ as well.

Proof. (a) Let $\epsilon$ be the parallel from $C$ to the line $A D$. Since $\epsilon$ does not intersect the line $A D$ it has to totally lie at one of the half-planes with respect to the line $A D$ say at $(\pi)$.

Now since $A B C D$ is convex, it totally lies at the same half plane with respect to the line $A D$. But as already mentioned the point $C$ of $A B C D$ lie with respect to the line $A D$ at the half-plane $(\pi)$. Thus $A B C D$ lies totally at $(\pi)$.

Let us call $C^{\prime}$ the intersection of the lines $A B, \epsilon . C^{\prime}$ indeed exists and is unique since $A B$ and the parallel $A D$ of $\epsilon$ intersect at a unique point; namely at $A$. Moreover, since $C^{\prime} \in \epsilon \subset(\pi), C^{\prime}$ lies at the halfline $A x=A D \cap(\pi)$. If it were true that $B$ was a point of $C^{\prime} x-\left\{C^{\prime}\right\}$ (Fig. 10b) then $A \widehat{D} C+B \widehat{C} D>A \widehat{D} C+D \widehat{C} C^{\prime}=180^{\circ}$ a contradiction by assumption.

So $B$ is a point of the segment $A C^{\prime}$.

Let $\epsilon^{\prime}$ be the parallel from $B$ to $A D$. $\epsilon^{\prime}$ intersects the line $A C$ at a unique point, say $B^{\prime}$, since its parallel line $A D$ does so. Since also $A D\left\|\epsilon^{\prime}\right\| \epsilon$ and $B$ is a point of the segment $A C^{\prime}, B^{\prime}$ has to be a point of the segment $D C$.

If $A B$ is parallel to $D C$ (Fig. 11a) then $A B B^{\prime} D$ is a parallelogram, so $\overrightarrow{B B^{\prime}}=\overrightarrow{A D}$ and the point $T$ is the point $B^{\prime}$ which lies on the side $D C$ of $A B C D$.

If $A B$ is not parallel to $D C$ (Fig. 11b) then their intersection point $O$ lies on the half-lines $B A, C D$ because of the relation $A \widehat{B} C+$ $B \widehat{C} D \leq 180^{\circ}$. Since $B^{\prime}$ is a point of the segment $D C$, the point $O$ lies on the half-lines $B A, B^{\prime} D$ as well. But Thales Theorem implies then $\frac{B B^{\prime}}{A D}=\frac{B O}{A O}>1 \Longrightarrow B B^{\prime}>A D$. This says that there exists a point $T^{\prime}$ on $B B^{\prime}$ such that $B T^{\prime}=A D$. Notice that since $A B C D$ is convex, the points $D, B^{\prime}$ of the side $D C$ lie on the same half-plane with respect to the line $A B$. So $B T^{\prime}=A D$ translates to $\overrightarrow{B T^{\prime}}=\overrightarrow{A D}$, 
which means that $T$ coincides with $T^{\prime}$ i.e. an interior point of the segment $B B^{\prime}$. Finally, since $A B C D$ is convex, and $B$ belongs to side $A B$ and $B^{\prime}$ belongs to side $D C$, all interior points of the segment $B B^{\prime}-T$ is one of them- lie in the interior of $A B C D$.

Now since $A B C D$ is convex, its diagonal $B D$ is internal, partitioning $\overline{A B C D}$ into the two triangular regions $\overline{A D B}, \overline{D B C}$. Because of this partitioning, one would have that $T$ is a point of $\overline{D B C}$ if he/she could prove that $T$ is not a point of $\overline{A D B}$.

But $\overrightarrow{B T}=\overrightarrow{A D} \Longrightarrow A B T D=$ parallelogram $=$ convex polygon $\Longrightarrow T, A$ lie in different half-planes with respect to the line $D B$. So $T$ cannot lie in $\overline{A D B}$.

Necessarily then, $T$ is a point of $\overline{D B C}$. Since $T$ is an interior point of $A B C D$ it cannot lie on the segments $B C, C D$. But $T$ cannot either lie on $D C$ since otherwise the line $B T$ would coincide with the line $B D$ thus intersecting the line $A D$, a contradiction by the fact that line $B T$ is parallel to $A D$. We conclude that $T$ is an interior point of $D B C$.

(b) Note that $\overrightarrow{B T}=(\overrightarrow{A D}=) \overrightarrow{M C}$. Thus:

If $T$ does not lie on the line $B C$ then $B T C M$ is a genuine parallelogram (Fig. 11b), and then the points $T, M$ lie on different half-planes with respect to the line $B C$. Since the $A B C D$ and its interior lie at the same side with respect to the line $B C$ as does the interior point $T$, we conclude that $M$ has to lie at the other half-plane, and so definitely at the exterior of $A B C D$.

If $T$ lies on the line $B C$, then $M$ also does. Note that $T$ lies on the line $B C$ iff $A D \| B C$ (Fig. 11c). Moreover then:

- In the case $A B$ is not parallel to $D C$, we proved in (a) that $B B^{\prime}>$ $A D$ which in our case translates to $B C>A D=C M$, and so $M$ is actually a point of the segment $B C$.

- In the case $A B$ is parallel to $D C$, pairing it with $A D \| B C$ we conclude that $A B C D$ is a parallelogram, thus $M$ coincides with $B$ which of course is a point of $B C$.

(c) In the case $(A B\|D C, A D\| B C)$ (Fig. 12a), the point $M$ coincides with $B$ and $A B M C$ becomes the triangle $A B C$.

In the case $A D \nVdash B C$, we proved in (b) that the points $M$ and $A$ (which is a point of $A B C D$ ) lie in different half-planes with respect to the line $B C$. Thus $A B M C$ is indeed a polygon.

- If moreover $A B \| D C$ (Fig. 12b) then the point $M$ lies on the line $A B$ and $A B M C$ is not a genuine quadrilateral but a triangle. 
- If instead $A B \nVdash D C$ (Fig. 12c) we will show that the segments $A M, B C$ intersect at an interior point for each of them, thus $A B M C$ would be a convex (genuine) quadrilateral, and we will be done. Indeed:

We proved in (a) that in this case there exists a point $B^{\prime}$ on the segment $B C$ such that $B B^{\prime} \| A D$ and $B B^{\prime}>A D$. Since $B B^{\prime}>A D$ there exist an interior point $M^{\prime}$ of $B B^{\prime}$ such that $B^{\prime} M^{\prime}=A D$. Since $M^{\prime}$ is an interior point of $B B^{\prime}$ and $B, B^{\prime}$ are points on the convex quadrilateral $A B C D, M^{\prime}$ is moreover an interior point of $A B C D$. Then we know that the points $A, M^{\prime}$ lie on the same half-plane with respect to the line $D B^{\prime}$ (i.e. the line $D C$ ). But then $B^{\prime} M^{\prime}=A D$ gives $\overrightarrow{B^{\prime} M^{\prime}}=\overrightarrow{A D}=\overrightarrow{C M}$ which means that the point $M^{\prime}$ lies on the line $A M$. Since $M^{\prime}$ is an interior point of $A B C D$ and $M$ is an exterior point, the line $M^{\prime} M$ intersects $A B C D$ in exactly two points. One of them is $A$. Let $M^{\prime \prime}$ be the other one.

Of course $M^{\prime \prime}$ cannot lie on $A B$ for then line $A B$ would have to coincide with line $A M$ which is parallel to $D C$, thus $A B \| D C$, a contradiction.

Also, $M^{\prime \prime}$ cannot lie on $A D$ for then line $A D$ would have to coincide with line $A M$ which is parallel to $D C$, thus $A D \| D C$, a contradiction.

But $M^{\prime \prime}$ cannot lie on the side $D C$ either since $M^{\prime \prime} \in A M \| D C$.

We necessarily then have that $M^{\prime \prime} \in B C$ and that moreover it is an interior point of this segment.

Finally note that the points $A, M$ of the line $M^{\prime} M$ lie on different half-planes with respect to the line $B C$. So then, the point $M^{\prime \prime}$ at which the line $M^{\prime} M$ intersects the line $B C$ has to be an interior point of the segment $A M$.

The following lemma deals with areas and it is a well known result with a plethora of proofs.

Lemma 7. Let $A B C D$ be a convex quadrilateral, $K, N, L, M$ be the midpoints of the sides $A B, B C, C D, D A$ respectively, and $O$ be the intersection point of the segments $K L, M N$. Then (a) $K L, M N$ bisect each other, (b) $(A K O M)+(C L O M)=(B K O N)+(D L O M)$.

Proof. (a) Since $M, K$ are the midpoints of the segments $A D, A B$ we have $\overrightarrow{M K}=\frac{\overrightarrow{D B}}{2}$. similarly $\overrightarrow{L N}=\frac{\overrightarrow{D B}}{2}$. Thus $\overrightarrow{M K}=\overrightarrow{L N}$ and so $M K N L$ is a parallelogram, which implies the result. 


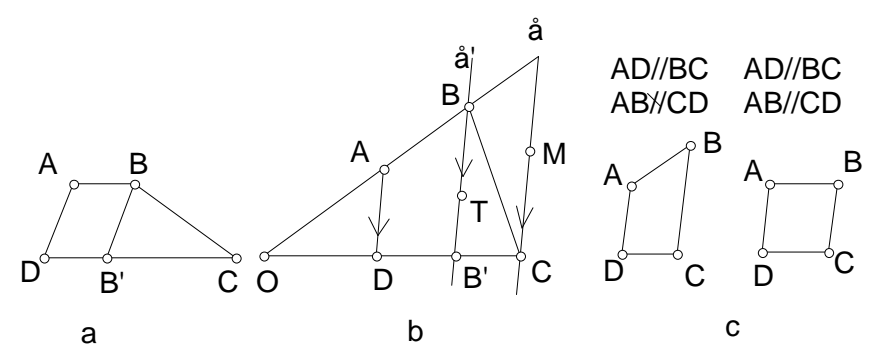

FIGURE 11

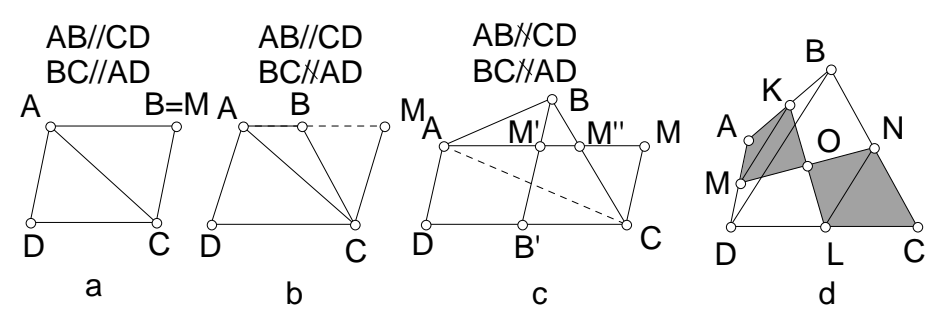

FiguRe 12

(b) It is (Fig. 12d)

$(A K O M)+(C L O M)=(A K M)+(O K M)+(C L N)+(O L N)$

Note that since $M, K$ are the midpoints of $A D, A B$ it is true that

$$
(A K M)=\frac{1}{4}(A B D) \text { and } \overrightarrow{K M}=\frac{1}{2} \overrightarrow{B D}
$$

Similarly,

$$
(C L N)=\frac{1}{4}(C B D) \text { and } \overrightarrow{N L}=\frac{1}{2} \overrightarrow{B D}
$$

(2.2), (2.3) imply

$$
(A K M)+(C L N)=\frac{1}{4}(A B D)+\frac{1}{4}(C B D)=\frac{1}{4}(A B C D)
$$


and $\overrightarrow{K M}=\overrightarrow{N L}$ thus $K N L M$ is a parallelogram, and so $(O K M)=$ $\frac{1}{4}(K N L M)=(O L N)$, from which

$$
(O K M)+(O L N)=\frac{1}{2}(K N L M)
$$

$(2.1),(2.4),(2.5) \Longrightarrow(A K O M)+(C L O M)=\frac{1}{4}(A B C D)+\frac{1}{2}(K N L M)$ A similar argument shows that $(B K O N)+(D L O M)=\frac{1}{4}(A B C D)$ $+\frac{1}{2}(K N L M)$ as well, establishing the result.

Our last lemma deals with the only property of long determinants needed in Section 3.

Lemma 8. The value of the long determinant

$$
\mid \begin{array}{ccccccc}
c_{1} & c_{2} & c_{3} & \cdots & c_{k} & & \\
& a_{1} & a_{2} & \cdots & a_{k-1} & a_{k} & a_{1} \\
& b_{1} & b_{2} & \cdots & b_{k-1} & b_{k} & b_{1} \\
d_{1} & d_{2} & d_{3} & \cdots & d_{k} & &
\end{array}
$$

all indices replacing $(1,2, \cdots k-1, k)$ by the same cyclic permutation simultaneously in all rows.

Proof. It is enough to show it whenever the indices $(1,2, \cdots, k-1, k)$ are replaced by their cyclic permutation $(2,3, \cdots, k, 1)$ :

$$
\begin{aligned}
& \text { It is } \\
& \left|\begin{array}{cccccccc}
c_{1} & c_{2} & c_{3} & \cdots & c_{k-1} & c_{k} & & \\
& a_{1} & a_{2} & \cdots & a_{k-2} & a_{k-1} & a_{k} & a_{1} \\
& b_{1} & b_{2} & \cdots & b_{k-2} & b_{k-1} & b_{k} & b_{1} \\
d_{1} & d_{2} & d_{3} & \cdots & d_{k-1} & d_{k} & &
\end{array}\right|= \\
& =\left(\sum_{i=1}^{k-1} c_{i} a_{i} b_{i+1}+c_{k} a_{k} b_{1}\right)-\left(\sum_{i=1}^{k-1} d_{i} b_{i} a_{i+1}+d_{k} b_{k} a_{1}\right) \\
& \text { and } \\
& \left|\begin{array}{cccccccc}
c_{2} & c_{3} & c_{4} & \cdots & c_{k} & c_{1} & & \\
& a_{2} & a_{3} & \cdots & a_{k-1} & a_{k} & a_{1} & a_{2} \\
& b_{2} & b_{3} & \cdots & b_{k-1} & b_{k} & b_{1} & b_{2} \\
d_{2} & d_{3} & d_{4} & \cdots & d_{k} & d_{1} & &
\end{array}\right|= \\
& =\left(\sum_{i=2}^{k-2} c_{i} a_{i} b_{i+1}+c_{k} a_{k} b_{1}+c_{1} a_{1} b_{2}\right)-\left(\sum_{i=2}^{k-2} d_{i} b_{i} a_{i+1}+d_{k} b_{k} a_{1}+\right. \\
& \left.d_{1} b_{1} a_{2}\right)=\left(\sum_{i=1}^{k-1} c_{i} a_{i} b_{i+1}+c_{k} a_{k} b_{1}\right)-\left(\sum_{i=1}^{k-1} d_{i} b_{i} a_{i+1}+d_{k} b_{k} a_{1}\right)
\end{aligned}
$$




\section{Proofs of the Main Results}

Proposition 1. Let $A B C D$ be a convex quadrilateral. If we divide each one of the sides $A B, D C$ in $m$ equal parts, and each of the sides $A D, B C$ in $n$ equal parts $\left(m, n \in \mathbb{N}^{*}\right)$, then by joining the corresponding division points of opposite sides by line segments, each of these segments from $A B$ to $D C$ is divided into $n$ equal parts, while each of these segments from $A D$ to $B C$ is divided into $m$ equal parts.

Thus a convex distorted lattice $L_{A B C D}\{m, n\}$ is formed.

Proof. Let us call each of the segments from $A B$ to $D C$ as vertical, and each of the segments from $A D$ to $B C$ as horizontal.

Let us also call our proposition as $P(n, m)$. We are going to use a double-induction argument.

$P(n, 1)$ is trivially true: The partition points of the segments $A B, D C$ are just $\{A, B\}$ and $\{D, C\}$ respectively; so the only vertical segments are $A D, B C$. This implies that the two vertical segments intersect each horizontal segment only at its two endpoints and so each horizontal segment is "partitioned" in just one part. Of course by assumption the horizontal segments partition each one of $A D, B C$ i.e. the vertical segments, in $n$ equal parts.

We are now going to prove that $P(n, 2)$ is true for all $n \geqq 1$. We achieve this by induction on $n$ :

For $n=1$ it is a most trivially true proposition. Just imitate the proof of $P(n, 1)$.

For $n=2$ we met it before as Lemma 7a. Let us suppose that $P(n, 2)$ holds for some $n \geqq 2$.

Then for $P(n+1,2)$ :

Let the partition points of $A D$ and $B C$ into $n+1$ equal parts be $A=L_{00}, L_{01}, L_{02}, \cdots, L_{0 n}, L_{0(n+1)}=D$, and $B=L_{10}, L_{11}, L_{12}$, $\cdots, L_{1 n}, L_{1(n+1)}=C$ respectively. Let also the midpoints of the segments $L_{00} L_{10}, L_{01} L_{11}, \cdots, L_{0(n+1)} L_{1(n+1)}$ be $L_{0}, L_{1}, \cdots, L_{n+1}$ (Fig. 13a).

Note now that the quadrilateral $L_{00} L_{10} L_{1 n} L_{0 n}$ is convex because of Lemma 5. Then applying the true proposition $P(n, 2)$ to the quadrilateral $L_{00} L_{10} L_{1 n} L_{0 n}$ we get that the line $L_{0} L_{n}$ contains the points $L_{0}, L_{1}, \cdots, L_{n-1}, L_{n}$.

Similarly, applying the true proposition $P(n, 2)$ to the (convex because of Lemma 5) quadrilateral $L_{01} L_{11} L_{1(n+1)} L_{0(n+1)}$ we get that the line $L_{1} L_{n+1}$ contains the points $L_{1}, L_{2}, \cdots, L_{n}, L_{n+1}$. 
Since $n \geqq 2$, the two points $L_{1}, L_{2}$ lie on both lines $L_{0} L_{n}, L_{1} L_{n+1}$. Thus these lines coincide and contain all $L_{0}, L_{1}, \cdots, L_{n-1}, L_{n}, L_{n+1}$. This proves that $P(n+1,2)$ is true.

Next, we are going to prove that for a given $n \geqq 1$, the proposition $P(n, m)$ is true for all $m \geqq 2$. We achieve this by induction on $m$ :

So let us fix a value greater or equal to 1 for $n$. Then:

For $m=1$, our proposition becomes proposition $P(n, 1)$ which we have already proved above.

For $m=2$, our proposition becomes proposition $P(n, 2)$ which we have also proved above. Let us suppose that $P(n, m)$ is true for some $m \geqq 2$. Then for $P(n, m+1)$ :

Let the partition points of $A B, D C$ into $m$ equal parts (Fig. 13b) be respectively

$$
\begin{aligned}
& A=L_{00}, L_{10}, L_{20}, \cdots, L_{m 0}, L_{(m+1) 0}=B \text { and } \\
& D=L_{0 n}, L_{1 n}, L_{2 n}, \cdots, L_{m n}, L_{(m+1) n}=C, \\
& \text { and let the partition points of } A D, B C \text { into } n \text { equal parts be } \\
& A=L_{00}, L_{01}, L_{02}, \cdots, L_{0(n-1)}, L_{0 n}=D \text { and } \\
& B=L_{(m+1) 0}, L_{(m+1) 1}, L_{(m+1) 2}, \cdots, L_{(m+1)(n-1)}, L_{(m+1) n}=C .
\end{aligned}
$$

Let finally the partition points of the segments $L_{10} L_{1 n}, L_{m 0} L_{m n}$ into $n$ equal parts be respectively

$$
\begin{aligned}
& L_{10}, L_{11}, \cdots, L_{1(n-1)}, L_{1 n} \text { and } \\
& L_{m 0}, L_{m 1}, \cdots, L_{m(n-1)}, L_{m n} .
\end{aligned}
$$

Note now that the quadrilateral $L_{00} L_{m 0} L_{m n} L_{0 n}$ is convex because of Lemma 5 Then applying the true proposition $P(n, m)$ to this quadrilateral we get that the lines $L_{00} L_{m 0}, L_{01} L_{m 1}, \cdots, L_{0 n} L_{m n}$ contain respectively the $0^{t h}, 1^{\text {st }}, 2^{\text {nd }}, \cdots, n^{\text {th }}$ partition points in $n$ equal parts of all the segments $c_{0}=L_{00} L_{0 n}, c_{1}=L_{10} L_{1 n}, c_{2}=$ $L_{20} L_{2 n}, \cdots, c_{m}=L_{m 0} L_{m n}$.

Similarly, applying the true proposition $P(n, m)$ to the (convex because of Lemma 5) quadrilateral $L_{10} L_{(m+1) 0} L_{(m+1) n} L_{1 n}$ we get that the lines $L_{10} L_{(m+1) 0}, L_{11} L_{(m+1) 1}, \cdots, L_{1 n} L_{(m+1) n}$ contain respectively the $0^{t h}, 1^{s t}, 2^{\text {nd }}, \cdots, n^{\text {th }}$ partition points in $n$ equal parts of all the segments $c_{1}=c_{1}=L_{10} L_{1 n}, c_{2}=L_{20} L_{2 n}, \cdots$, $c_{m}=L_{m 0} L_{m n}, c_{m+1}=L_{(m+1) 0} L_{(m+1) n}$.

Then for each $i \in\{0,1,2, \cdots, n\}$ the $i^{t h}$ partition points of the two distinct (since $m \geqq 2$ ) segments $c_{1}=L_{10} L_{0 n}, c_{2}=L_{20} L_{2 n}$ lie on both lines $L_{0 i} L_{m i}, L_{1 i} L_{(m+1) i}$. Thus for each $i \in\{0,1,2, \cdots, n\}$ these lines coincide and contain the $i^{t h}$ partition points of all $c_{0}, c_{1}$, $\cdots, c_{m}, c_{m+1}$. This proves that $P(n, m+1)$ is true. 


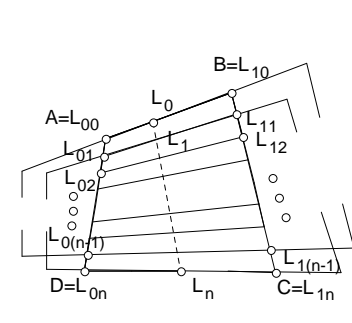

a

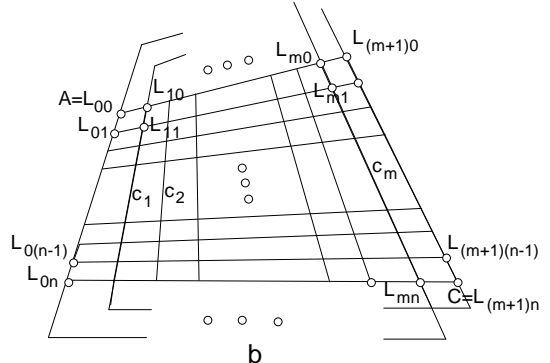

b

Figure 13

Corollary 1. If we divide each one of two opposite sides of a convex quadrilateral region into $2 n$ equal parts and each one of the other two opposite sides into $2 m$ equal parts $\left(n, m \in N^{*}\right)$, then connect the corresponding division points of the opposite sides by line segments and finally colour the quadrilateral regions formed as black and white in a chess like manner (i.e. any two regions sharing a common side are differently painted), then it is true that the total black area equals the total white area.

Proof. If $A B C D$ is our quadrilateral, we know by the previous proposition that by dividing its sides as stated, it is formed a convex distorted lattice $L_{A B C D}\{2 m, 2 n\}$ and by Lemma 5 that $\overline{A B C D}$ is partitioned into a number of convex quadrilateral regions $\overline{P_{k}}$. We group these quadrilaterals by 4 as in the Figure 14a, i.e. we form the larger quadrilateral regions $\overline{P_{i j}}=\overline{L_{(2 i)(2 j)} L_{(2 i+2)(2 j)} L_{(2 i+2)(2 j+2)} L_{(2 i+2)(2 j)}}$ $i \in\{0,2(n-1)\}, j \in\{0,2(m-1)\}$.

In each $\overline{P_{i j}}$, the lattice points on their sides are just the endpoints and midpoints of the sides. Again by Lemma 5 we know that each $\overline{P_{i j}}$ is convex. So then Lemma $7 \mathrm{~b}$ implies that the two black $\overline{P_{k}}$ 's contained in $\overline{P_{i j}}$ have total area equal to the total area of the two white $\overline{P_{k}}$ 's contained in $\overline{P_{i j}}$. So by adding for all $i, j$, we get that the sum of all $\overline{P_{k}}$ with black area equals the sum of all $\overline{P_{k}}$ with white area.

The next seven lemmas are special cases of our main theorem and they lead us gradually to the theorem itself. 


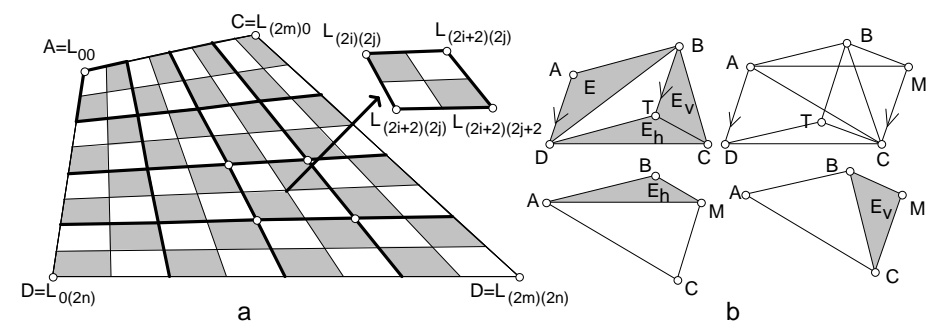

FIGURE 14

Lemma 9. Let $A B C D$ be a convex quadrilateral with interior angles such that $\widehat{B}+\widehat{C} \leq 180^{\circ}, \widehat{C}+\widehat{D} \leq 180^{\circ}$. Let $T$ be a point such that $\overrightarrow{B T}=\overrightarrow{A D}$ and let us call $(A B D)=E,(B T C)=E_{v},(D T C)=E_{h}$. Then $(D B C)=E+E_{h}+E_{v},(A B C)=E+E_{h},(A C D)=E+E_{v}$.

Proof. We will prove it in the hardest case $\widehat{B}+\widehat{C}<180^{\circ}, \widehat{C}+\widehat{D}<$ $180^{\circ}$. All other cases are just limit cases of this "hard" one and the proof demands only trivial alterations of the one we give below (or since then at least one pair of opposite sides of $A B C D$ are parallel, many other even easier proofs can be given).

About $(D B C)$ :

By Lemma 6 we have that $T$ is an interior point of $D B C$ (Fig. 14b), so then $(D B C)=(D B T)+(B T C)+(D T C) \Longrightarrow$

$$
(D B C)=(D B T)+E_{h}+E_{v}
$$

But $\overrightarrow{B T}=\overrightarrow{A D} \Longrightarrow A B T D=$ parallelogram $\Longrightarrow$

$$
(D B T)=(A B D)=E
$$

(3.1) $(3.2) \Longrightarrow(D B C)=E+E_{h}+E_{v}$ as wanted.

About $(A B C)$ and $(A C D)$ :

First observe that $(A B C D)=(A B C)+(A C D)$ thus

$$
(A B C)+(A C D)=2 E+E_{h}+E_{v}
$$

Let us now consider a point $M$ such that $\overrightarrow{M C}=\overrightarrow{A D}$. We know by Lemma 6 that $M$ is an exterior point of $A B C D$ (Fig. 14b) and that $A B M C$ is convex.

Let us observe that $\overrightarrow{B T}=\overrightarrow{A D}=\overrightarrow{M C} \Longrightarrow B M C T$ is a parallelogram, thus

$$
\overrightarrow{B M}=\overrightarrow{T C}
$$




$$
\text { and }(B M C)=(B T C)=E_{v}
$$

Similarly $\overrightarrow{A D}=\overrightarrow{M C} \Longrightarrow D A M C=$ parallelogram, so

$$
(A M C)=(A C D)
$$

Note that we have shown above $A B T D$ to be a parallelogram, and so we have

$$
\overrightarrow{B A}=\overrightarrow{T D}
$$

(3.4), (3.7) $\Longrightarrow$ triangle $A B M=$ triangle $D T C \Longrightarrow$

$$
(A B M)=(D T C)=E_{h}
$$

Now since $A B M C$ is convex its diagonals are internal and we have

$$
\begin{gathered}
(A B M C)=(A B M)+(A M C)=(A B C)+(B M C) \stackrel{(3.5),(3.6),(3.8)}{\longrightarrow} \\
E_{h}+(A C D)=(A B C)+E_{v}
\end{gathered}
$$

Solving the system of (3.3), (3.9) we get $(A B C)=E+E_{h},(A C D)$ $=E+E_{v}$ as wanted (Fig. 15a).

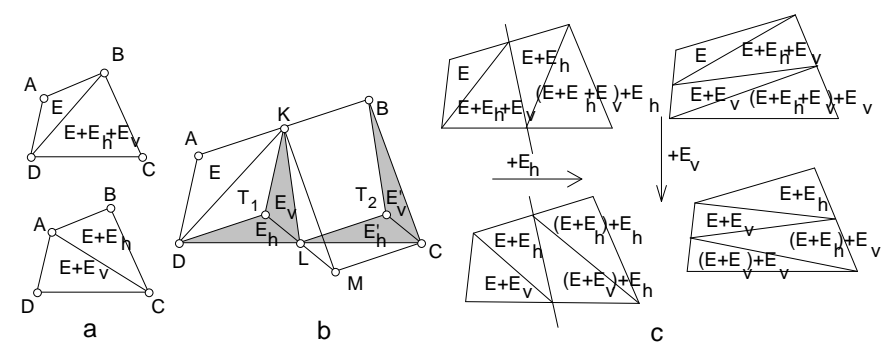

FIGURE 15

Lemma 10. Let $A B C D$ be a convex quadrilateral with interior angles such that $\widehat{B}+\widehat{C} \leq 180^{\circ}, \widehat{C}+\widehat{D} \leq 180^{\circ}$ and $K, L$ be the midpoints of the sides $A B, C D$ respectively. Let also $T_{1}, T_{2}$ be points such that $\overrightarrow{K T}_{1}=\overrightarrow{A D}, \overrightarrow{B T}_{2}=\overrightarrow{K L}$, and let us call $(A K D)=E,\left(K T_{1} L\right)=$ $E_{v},\left(D T_{1} L\right)=E_{h}$ and $(K B L)=E^{\prime},\left(B T_{2} C\right)=E_{v}^{\prime},\left(L T_{2} C\right)=E_{h}^{\prime}$. Then

$E^{\prime}=E+E_{h}, E_{h}^{\prime}=E_{h}, E_{v}^{\prime}=E_{v},(K B L)=E+E_{h}=(A K D)+$ $E_{h},(L B C)=E+2 E_{h}+E_{v}=(D K L)+E_{h},(K B C)=E+2 E_{h}=$ $(A K L)+E_{h},(L K C)=E+E_{h}+E_{v}=(D A L)+E_{h}$. 
In other words, as we move from the triangles of $A K L D$ (formed by its diagonals) to the right at the corresponding triangles of $K B C L$ (formed by its diagonals) the areas increase by $E_{h}$ (Fig. 15c).

Proof. By Lemmas 2,4 we know that $A K L D, K B C L$ are convex and that for their interior angles the following hold respectively: $\left(\widehat{K}+\widehat{L} \leq 180^{\circ}, \widehat{L}+\widehat{D} \leq 180^{\circ}\right),\left(\widehat{B}+\widehat{C} \leq 180^{\circ}, \widehat{C}+\widehat{L} \leq 180^{\circ}\right)$.

Then applying Lemma 9 to $A K L D, K B C L$ we get respectively

$$
\begin{gathered}
(A K D)=E,(D K L)=E+E_{h}+E_{v},(A K L)=E+E_{h} \\
(D A L)=E+E_{v} \\
(K B L)=E^{\prime},(L B C)=E^{\prime}+E_{h}^{\prime}+E_{v}^{\prime},(K B C)=E^{\prime}+E_{h} \\
(L K C)=E^{\prime}+E_{v}^{\prime}
\end{gathered}
$$

Thus we would have proved all the required relations if we could only establish the first three of them: $E^{\prime}=E+E_{h}, E_{h}^{\prime}=E_{h}, E_{v}^{\prime}=E_{v}$. This is not hard to do:

(a) Observe that $L$ is the midpoint of the side $A B$ of the triangle $L A B$. Thus $(K B L)=(A K L)$, which because of $(3.10),(3.11)$ becomes $E^{\prime}=E+E_{h}$.

(b) Let us consider the point $M$ defined by $\overrightarrow{C M}=\overrightarrow{B K}$ (Fig. 15b).

Then $\overrightarrow{C M}=\overrightarrow{B K}=\overrightarrow{K A}=\overrightarrow{T_{2} L}$, where the last equality holds since $A K T_{1} D$ is a parallelogram (because of $\overrightarrow{K T}_{1}=\overrightarrow{A D}$ ).

So $M C D T_{1}$ is a parallelogram. Then the midpoint $L$ of the diagonal $D C$ is the center of the parallelogram and it is well known that $\left(D T_{1} L\right)=(L M C)$ i.e.

$$
E_{h}=(L M C)
$$

But, $\overrightarrow{B T_{2}}=\overrightarrow{K L} \Longrightarrow B T_{2} L K$ is a parallelogram $\Longrightarrow \overrightarrow{B K}=\overrightarrow{T_{2} L} \Longrightarrow$ $\overrightarrow{C M}=\overrightarrow{T_{2} L} \Longrightarrow C M L T_{2}$ is a parallelogram, thus

$$
(L M C)=\left(L T_{2} C\right)=E_{h}^{\prime}
$$

(3.12), (3.13) $\Longrightarrow E_{h}^{\prime}=E_{h}$.

(c) $\overrightarrow{C M}=\overrightarrow{B K}$ imply that $B C M K$ is a parallelogram and so $\overrightarrow{K L}=\overrightarrow{B C}$. This, paired with $\overrightarrow{B T_{2}}=\overrightarrow{K L}$ imply equality for the triangles $K L M, B T_{2} C$. Consequently $(K L M)=\left(B T_{2} C\right)$ i.e.

$$
(K L M)=E_{v}^{\prime}
$$


But note that we have just shown in (b) that $L$ is the center of the parallelogram $M C D T_{1}$. Then $L$ is also the midpoint of the segment $T_{1} M$. Thus $(K L M)=\left(K L T_{1}\right)$ i.e.

$$
(K L M)=E_{v}
$$

(3.14), (3.15) $\Longrightarrow E_{v}^{\prime}=E_{v}$.

Similarly we can prove

Lemma 11. Let $A B C D$ be a convex quadrilateral with interior angles such that $\widehat{B}+\widehat{C} \leq 180^{\circ}, \widehat{C}+\widehat{D} \leq 180^{\circ}$ and let $M, N$ be the midpoints of the sides $A D, B C$ respectively. Let also $T_{1}, T_{2}$ be points such that $\overrightarrow{K T}_{1}=\overrightarrow{A B}, \overrightarrow{D T}_{2}=\overrightarrow{M N}$, and let us call $(A B M)=$ $E,\left(B T_{1} N\right)=E_{v},\left(M T_{1} N\right)=E_{h}$ and $(M N D)=E^{\prime},\left(N T_{2} C\right)=$ $E_{v}^{\prime},\left(D T_{2} C\right)=E_{h}^{\prime}$. Then

$E=E^{\prime}, E_{v}=E_{v}^{\prime}, E_{h}=E_{h}^{\prime},(M N D)=E+E_{v}=(A B M)+E_{v}$, $(D N C)=E+E_{h}+2 E_{v}=(M B N)+E_{h},(N C M)=E+E_{h}+E_{v}=$ $(B N A)+E_{v},(D M C)=E+2 E_{v}=(M A N)+E_{v}$.

In a certain sense this lemma is a restatement of the previous one. It essentially says that as we move from the triangles of $A D M N$ (formed by its diagonals) down at the corresponding triangles of $M N C D$ (formed by its diagonals) the areas increase by $E_{v}$ (Fig. 15c).

Lemma 12. Let us consider a convex distorted lattice $L_{A B C D}\{m, n\}$ and let us assume without loss of generality that for the interior angles of $A B C D$ it is $\widehat{B}+\widehat{C} \leq 180^{\circ}, \widehat{C}+\widehat{D} \leq 180^{\circ}$. Let $T$ be a point such that $\overrightarrow{L_{10} T}=\overrightarrow{L_{00} L_{01}}$. Equivalently $T$ is defined by $\overrightarrow{L_{01} T}=$ $\overrightarrow{L_{00} L_{10}}$. Let us call $\left(L_{01} L_{00} L_{10}\right)=E,\left(L_{10} T L_{11}\right)=E_{v},\left(L_{01} T L_{11}\right)=$ $E_{h}$. Then the areas of the triangles with vertices three "neighbor" lattice points are given by $\left(L_{(a-1) b} L_{a b} L_{a(b-1)}\right)=\left(L_{(a+1) b} L_{a b} L_{a(b+1)}\right)$ $=\left(L_{(a+1) b} L_{a b} L_{a(b+1)}\right)=\left(L_{(a+1) b} L_{a b} L_{a(b+1)}\right)=E+a E_{h}+b E_{v}$, whenever the triangles make sense.

Proof. Let us call $\bar{L}^{a b}=\overline{L_{a b} L_{(a+1) b} L_{(a+1)(b+1)} L_{a(b+1)}}, a \in\{0,1$, $\cdots, m-1\}, b \in\{0,1, \cdots, n-1\}$ the quadrilateral regions into which $A B C D$ is partitioned by the horizontal and the vertical lattice segments. By Lemma 4 we know that the union of any two of $\bar{L}^{a b}$ sharing a common side is a nicely positioned convex polygonal region. This allows us to apply the last two lemmas to any union of two $\bar{L}^{a b}$, s sharing a common side. The crucial point is to notice that 
since $T$ is defined by both $\overrightarrow{L_{10} T}=\overrightarrow{L_{00} L_{01}}, \overrightarrow{L_{01} T}=\overrightarrow{L_{00} L_{10}}$ then as we move to triangles horizontally to the right, their areas increase by $E_{h}$, while as we move to triangles vertically down, their areas increase by $E_{v}$ (Fig. 16). Then the required formula should be entirely clear. A mathematically rigorous proof can be given by an induction argument. Alternatively, the following heuristic argument is good enough for proof:

Let us define for each $\bar{L}^{a b}$ a point $T_{a b}$ such that $\overrightarrow{L_{(a+1) b} T_{a b}}=$ $\overrightarrow{L_{a b} L_{a(b+1)}}$ and let us call $\left(L_{a(b+1)} L_{a b} L_{(a+1) b}\right)=E^{a b}$,

$\left(L_{(a+1) b} T_{a b} L_{(a+1)(b+1)}\right)=E_{v}^{a b},\left(L_{a(b+1)} T_{a b} L_{(a+1)(b+1)}\right)=E_{h}^{a b}$.

Of course $T_{00}$ coincides with $T$, and $E^{00}=E, E_{v}^{00}=E_{v}, E_{h}^{00}=$ $E_{h}$.

Let us fix an index $a \in\{0,1, \cdots, m-1\}$.

Applying Lemma 10 successively to $\bar{L}^{00} \cup \bar{L}^{10}, \bar{L}^{10} \cup \bar{L}^{20}, \cdots$, $\bar{L}^{(a-1) 0} \cup \bar{L}^{a 0}$ we get the following interesting relations:

$$
E_{v}^{a^{\prime} 0}=E_{v}, E_{h}^{a^{\prime} 0}=E_{h}, \forall a^{\prime}=0,1, \cdots, a
$$

With these relations at hand, applying again Lemma 10 successively to $\bar{L}^{00} \cup \bar{L}^{10}, \bar{L}^{10} \cup \bar{L}^{20}, \cdots, \bar{L}^{(a-1) 0} \cup \bar{L}^{a 0}$ and recalling that $\left(L_{00} L_{10} L_{01}\right)=E$ we get:

$\left(L_{10} L_{20} L_{11}\right)=E+E_{h}$ and so then by $(3.16)$

$\left(L_{20} L_{30} L_{21}\right)=E+2 E_{h}$ and so then by $(3.16)$

:

$\left(L_{a 0} L_{(a+1) 0} L_{a 1}\right)=E+a E_{h}$.

Now we fix an index $b \in\{0, n-1\}$ and applying Lemma 10 successively to $\bar{L}^{a 0} \cup \bar{L}^{a 1}, \bar{L}^{a 1} \cup \bar{L}^{a 2}, \cdots, \bar{L}^{a(b-1)} \cup \bar{L}^{a b}$ we at least get

$$
E_{h}^{a b^{\prime}}=E_{h}, E_{v}^{a b^{\prime}}=E_{v}, \forall b^{\prime}=0,1, \cdots, b
$$

With these relations at hand we apply again Lemma 10 successively to $\bar{L}^{a 0} \cup \bar{L}^{a 1}, \bar{L}^{a 1} \cup \bar{L}^{a 2}, \cdots, \bar{L}^{a(b-1)} \cup \bar{L}^{a b}$ and recalling that $\left(L_{a 0} L_{(a+1) 0} L_{a 1}\right)=E+a E_{h}$ now we get:

$\left(L_{a 1} L_{(a+1) 1} L_{a 2}\right)=E+a E_{h}+E_{v}$ and so then

$\left(L_{a 2} L_{(a+1) 2} L_{a 3}\right)=E+a E_{h}+2 E_{v}$ and so then

$\left(L_{a b} L_{(a+1) b} L_{(a+1)(b+1)}\right)=E+a E_{h}+b E_{v}$

Observe that for the quadrilateral $L_{a(b-1)} L_{(a+1) b} L_{a(b+1)} L_{(a-1) b}$ (whenever it makes sense) its diagonals bisect each other. Thus the 
last relation gives

$$
\begin{gathered}
\left(L_{(a-1) b} L_{a b} L_{a(b-1)}\right)=\left(L_{(a+1) b} L_{a b} L_{a(b+1)}\right)=\left(L_{(a+1) b} L_{a b} L_{a(b+1)}\right)= \\
\left(L_{(a+1) b} L_{a b} L_{a(b+1)}\right)=E+a E_{h}+b E_{v}
\end{gathered}
$$

Since $a \in\{0, m-1\}, b \in\{0, n-1\}$, this proves the required result except for the triangles $L_{a n} L_{(a+1)(n-1)} L_{(a+1) n}, a \in\{0, m-1\}$. For these triangles we argue as follows:

By Lemma 5 we know that any $\bar{L}^{a(n-1)}, a \in\{0, m-1\}$ is a nicely positioned convex polygonal region. This allows us to apply Lemma 9 to it. Since (3.17) gives $E_{v}^{a(n-1)}=E_{v}, E_{h}^{a(n-1)}=E_{h}$ and (3.18) gives $\left(L_{(a+1)(n-1)} L_{a(n-1)} L_{a n}\right)=E+a E_{h}+(n-1) E_{v}$, applying Lemma 9 to $\bar{L}^{a(n-1)}$ we get $\left(L_{a n} L_{(a+1) n} L_{(a+1)(n-1)}\right)=$ $\left(L_{(a+1)(n-1)} L_{a(n-1)} L_{a n}\right)+E_{h}+E_{v}=\left[E+a E_{h}+(n-1) E_{v}\right]+E_{h}+$ $E_{v}=E+(a+1) E_{h}+n E_{v}$.

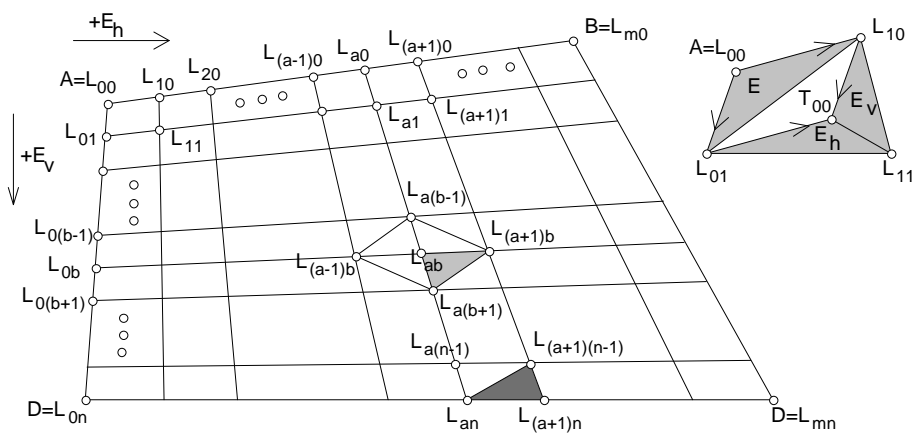

FiguRE 16

Lemma 13. Let us consider a convex distorted lattice $L_{A B C D}\{m, n\}$ and assume without loss of generality that for the interior angles of $A B C D$ it is $\widehat{B}+\widehat{C} \leq 180^{\circ}, \widehat{C}+\widehat{D} \leq 180^{\circ}$. Let $T$ be a point such that $\overrightarrow{L_{10} T}=\overrightarrow{L_{00} L_{01}}$. Equivalently $T$ is defined by $\overrightarrow{L_{01} T}=\overrightarrow{L_{00} L_{10}}$. Let us call $\left(L_{01} L_{00} L_{10}\right)=E,\left(L_{10} T L_{11}\right)=E_{v},\left(L_{01} T L_{11}\right)=E_{h}$. Then the areas of the triangles with one side on a lattice segment are given by

(a) $\left(L_{a_{1} b} L_{a_{2} b} L_{a_{3} b^{\prime}}\right)=\left|a_{2}-a_{1}\right|\left|b^{\prime}-b\right|\left(E+a_{3} E_{h}+b E_{v}\right)=$ 


$$
\begin{aligned}
& \pm\left(\left|\begin{array}{ccccc}
1 & 1 & 1 & & \\
& a_{1} & a_{2} & a_{3} & a_{1} \\
& b & b & b^{\prime} & b \\
1 & 1 & 1 & &
\end{array}\right| E+\left|\begin{array}{ccccc}
a_{2} & a_{3} & a_{1} & & \\
& a_{1} & a_{2} & a_{3} & a_{1} \\
& b & b & b & b^{\prime} \\
a_{1} & a_{2} & a_{3} & &
\end{array}\right| E_{h}+\right. \\
& \left.+\left|\begin{array}{ccccc}
b & b & b^{\prime} & & \\
& a_{1} & a_{2} & a_{3} & a_{1} \\
& b & b & b^{\prime} & b \\
b & b^{\prime} & b & &
\end{array}\right| E_{v}\right) \\
& \text { (b) }\left(L_{a b_{1}} L_{a b_{2}} L_{a^{\prime} b_{3}}\right)=\left|a^{\prime}-a\right|\left|b_{2}-b_{1}\right|\left(E+a E_{h}+b_{3} E_{v}\right)= \\
& \pm\left(\left|\begin{array}{ccccc}
1 & 1 & 1 & & \\
& a & a & a^{\prime} & a \\
& b_{1} & b_{2} & b_{3} & b_{1} \\
1 & 1 & 1 & &
\end{array}\right| E+\left|\begin{array}{ccccc}
a & a^{\prime} & a & & \\
& a & a & a & a^{\prime} \\
& b_{1} & b_{2} & b_{3} & b_{1} \\
a & a & a^{\prime} & &
\end{array}\right| E_{h}+\right. \\
& \left.+\left|\begin{array}{ccccc}
b_{1} & b_{2} & b_{3} & & \\
& a & a & a^{\prime} & a \\
& b_{1} & b_{2} & b_{3} & b_{1} \\
b_{2} & b_{3} & b_{1} & &
\end{array}\right| E_{v}\right)
\end{aligned}
$$

The + sign holds whenever the positive orientations in the plane of $L_{a_{1} b} L_{a_{2} b} L_{a_{3} b^{\prime}}$ and $L_{a b_{1}} L_{a b_{2}} L_{a^{\prime} b_{3}}$ are $\left(L_{a_{1} b}, L_{a_{2} b}, L_{a_{3} b^{\prime}}\right)$ and $\left(L_{a b_{1}}\right.$, $\left.L_{a b_{2}}, L_{a^{\prime} b_{3}}\right)$, respectively.

Proof. We will prove only (a) since part (b) is proved in a similar manner.

In order to facilitate notation we call $M=L_{a_{1} b}, N=L_{a_{2} b}, O=$ $L_{a_{3} b^{\prime}}, P=L_{a_{3} b}, G=L_{\left(a_{3}+1\right) b}, H=L_{a_{3}(b-1)}$ (Fig. 17a). We want to prove

$$
\begin{array}{r}
(O M N)=\left|a_{2}-a_{1}\right|\left|b^{\prime}-b\right|\left(E+a_{3} E_{h}+b E_{v}\right) . \\
\text { It is } \frac{O P}{H P}=\left|b^{\prime}-b\right| \Longrightarrow \frac{(O M N)}{(H M N)}=\left|b^{\prime}-b\right| \Longrightarrow \\
(O M N)=\left|b^{\prime}-b\right|(H M N)
\end{array}
$$

And $\frac{M N}{P G}=\left|a_{2}-a_{1}\right| \Longrightarrow \frac{(H M N)}{(H P G)}=\left|a_{2}-a_{1}\right| \Longrightarrow$

$$
(H M N)=\left|a_{2}-a_{1}\right|(H P G)
$$

But the previous lemma gives us

$$
(H P G)=E+a_{3} E_{h}+b E_{v}
$$

(3.19), (3.20), (3.21) $\Longrightarrow(O M N)=\left|a_{2}-a_{1}\right|\left|b^{\prime}-b\right|\left(E+a_{3} E_{h}+b E_{v}\right)$ as wanted. 
Note that whenever the indices are such that $G=L_{\left(a_{3}+1\right) b}$ or $H=L_{a_{3}(b-1)}$ is not defined (i.e. for $a_{3}=n$ or $b=0$ ), then the point $G^{\prime}=L_{\left(a_{3}-1\right) b}$ or $F^{\prime}=L_{a_{3}(b+1)}$ is defined and it can replace $G$ or $H$ in the above argument with no other changes.

Observe now that the orientation $\left(L_{a_{1} b}, L_{a_{2} b}, L_{a_{3} b^{\prime}}\right)$ of the triangle $L_{a_{1} b} L_{a_{2} b} L_{a_{3} b^{\prime}}$ is its positive orientation in the plane exactly when $\left(a_{1}<a_{2}\right.$ and $\left.b^{\prime}>b\right)$ or $\left(a_{1}>a_{2}\right.$ and $\left.b^{\prime}<b\right)$ (Fig. 17b). In these cases our formula becomes

$$
\left(L_{a_{1} b} L_{a_{2} b} L_{a_{3} b^{\prime}}\right)=\left(a_{2}-a_{1}\right)\left(b^{\prime}-b\right)\left(E+a_{3} E_{h}+b E_{v}\right)=\left(a_{2} b^{\prime}+\right.
$$
$\left.a_{1} b-a_{2} b-a_{1} b^{\prime}\right) E+\left(a_{3} a_{2} b^{\prime}+a_{3} a_{1} b-a_{3} a_{2} b-a_{3} a_{1} b^{\prime}\right) E_{h}+\left(b a_{2} b^{\prime}+\right.$ $\left.b a_{1} b-b a_{2} b-b a_{1} b^{\prime}\right) E_{v}$

$$
\begin{aligned}
& =\left|\begin{array}{ccccc}
1 & 1 & 1 & & \\
& a_{1} & a_{2} & a_{3} & a_{1} \\
& b & b & b^{\prime} & b \\
1 & 1 & 1 & &
\end{array}\right| E+\left|\begin{array}{ccccc}
a_{2} & a_{3} & a_{1} & & \\
& a_{1} & a_{2} & a_{3} & a_{1} \\
& b & b & b & b^{\prime} \\
a_{1} & a_{2} & a_{3} & &
\end{array}\right| E_{h}+ \\
& +\left|\begin{array}{ccccc}
b & b & b^{\prime} & & \\
& a_{1} & a_{2} & a_{3} & a_{1} \\
& b & b & b^{\prime} & b \\
b & b^{\prime} & b & &
\end{array}\right| E_{v}
\end{aligned}
$$

A similar argument shows that if $\left(L_{a_{1} b}, L_{a_{2} b}, L_{a_{3} b^{\prime}}\right)$ is the negative orientation of $L_{a_{1} b} L_{a_{2} b} L_{a_{3} b^{\prime}}$ in the plane then the - sign holds in the last equality of our formula.

Lemma 14. Let us consider a convex distorted lattice $L_{A B C D}\{m, n\}$ and let us assume without loss of generality that for the interior angles of $A B C D$ it is $\widehat{B}+\widehat{C} \leq 180^{\circ}, \widehat{C}+\widehat{D} \leq 180^{\circ}$. Let $T$ be a point such that $\overrightarrow{L_{10} T}=\overrightarrow{L_{00} L_{01}}$. Equivalently $T$ is defined by $\overrightarrow{L_{01} T}=$ $\overrightarrow{L_{00} L_{10}}$. Let us call $\left(L_{01} L_{00} L_{10}\right)=E,\left(L_{10} T L_{11}\right)=E_{v},\left(L_{01} T L_{11}\right)=$ $E_{h}$. Then the areas of the quadrilaterals with sides on the lattice segments are given by

$$
\left(L_{a c} L_{b c} L_{b d} L_{a d}\right)=|b-a||d-c|\left(2 E+(a+b) E_{h}+(c+d) E_{v}\right) .
$$

Observe that the orientation $\left(L_{a c}, L_{b c}, L_{b d}, L_{a d}\right)$ of $L_{a c} L_{b c} L_{b d} L_{a d}$ is its positive orientation in the plane exactly when $(a<b$ and $c<d)$ or $(a>b$ and $c>d)$. In this case the area of $L_{a c} L_{b c} L_{b d} L_{a d}$ is given by $\left(L_{a c} L_{b c} L_{b d} L_{a d}\right)=(b-a)(d-c)\left[2 E+(a+b) E_{h}\right]+(c+d) E_{v}$.

Proof. It is (Fig. 17c)

$$
\begin{aligned}
& \left(L_{a c} L_{b c} L_{b d} L_{a d}\right)=\left(L_{a c} L_{a d} L_{b d}\right)+\left(L_{a c} L_{b c} L_{b d}\right) \stackrel{\text { previous lemma }}{=} \\
& =|b-a||d-c|\left(E+a E_{h}+d E_{v}\right)+|b-a||d-c|\left(E+c E_{v}+b E_{h}\right)
\end{aligned}
$$


$=|b-a||d-c|\left[2 E+(a+b) E_{h}+(c+d) E_{v}\right]$.

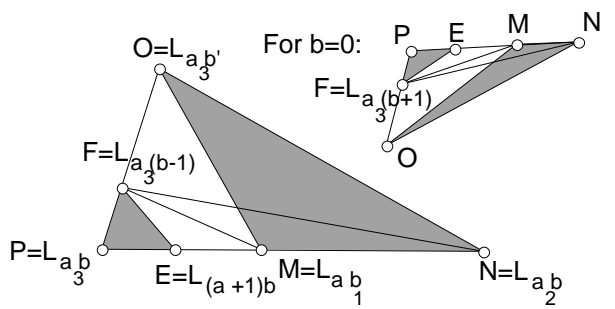

a

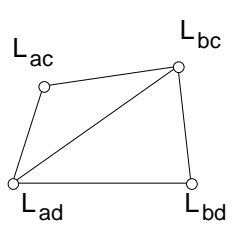

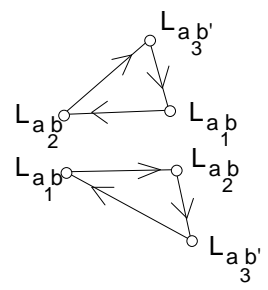

b

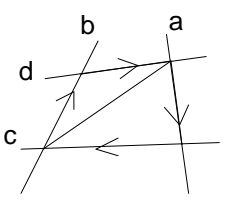

C

FiguRe 17

Lemma 15. Let us consider a convex distorted lattice $L_{A B C D}\{m, n\}$ and assume without loss of generality that for the interior angles of $A B C D$ it is $\widehat{B}+\widehat{C} \leq 180^{\circ}, \widehat{C}+\widehat{D} \leq 180^{\circ}$. Let $T$ be a point such that $\overrightarrow{L_{10} T}=\overrightarrow{L_{00} L_{01}}$ and let us call $\left(L_{01} L_{00} L_{10}\right)=E,\left(L_{10} T L_{11}\right)=$ $E_{v},\left(L_{01} T L_{11}\right)=E_{h}$. If $A_{1} A_{2} A_{3}$ is a lattice triangle with $A_{i}=$ $\left(a_{i}, b_{i}\right)=L_{a_{i} b_{i}}$ then

$$
\begin{aligned}
& \begin{array}{lll}
1 & 1 & 1
\end{array}
\end{aligned}
$$

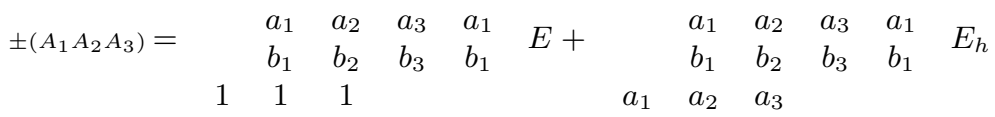

$$
\begin{aligned}
& \begin{array}{lll}
b_{1} & b_{2} & b_{3}
\end{array} \\
& +\quad \begin{array}{ccccc}
a_{1} & a_{2} & a_{3} & a_{1} & E_{v} \\
b_{1} & b_{2} & b_{3} & b_{1}
\end{array} \\
& \begin{array}{lll}
b_{2} & b_{3} & b_{1}
\end{array}
\end{aligned}
$$

The + sign holds exactly when $\left(A_{1}, A_{2}, A_{3}\right)$ is the positive orientation of $A_{1} A_{2} A_{3}$ in the plane. 
Proof. For $A_{1} A_{2} A_{3}$ with one side lying on a lattice segment, the result was shown in Lemma 13. So let us assume that no side of $A_{1} A_{2} A_{3}$ lies on any lattice segment.

We will treat the case when $\left(A_{1}, A_{2}, A_{3}\right)$ is the positive orientation of $A_{1} A_{2} A_{3}$ in the plane; the other case is proved similarly. Note that since the sides of $A_{1} A_{2} A_{3}$ do not lie on lattice segments, the first coordinates $a_{1}, a_{2}, a_{3}$ of its vertices are distinct, and similarly the second coordinates $b_{1}, b_{2}, b_{3}$ are distinct as well.

Note also that since $\left(A_{1}, A_{2}, A_{3}\right)$ is the positive orientation of $A_{1} A_{2} A_{3}$ in the plane, then by changing the indices in this orientation by a cyclic permutation of $(1,2,3)$ we end up with the same positive orientation of $A_{1} A_{2} A_{3}$ in the plane. But since by Lemma 8 the value of the long determinants do not change if the indices in each row change by the same cyclic permutation of $(1,2,3)$, we can assume that $a_{1}$ is the smallest number among $a_{1}, a_{2}, a_{3}$. Now since $b_{1}, b_{2}, b_{3}$ are distinct, all possible placements of $A_{1} A_{2} A_{3}$ on the lattice as shown in Figure 18.

So a way to achieving our goal is to prove the formula for each one of the cases of Figure 18.

We shall demonstrate it in only two cases; the method is the same for all of them, and the result comes similarly trivially in all cases. In a more conventional notation we wish to prove

$\left(A_{1} A_{2} A_{3}\right)=\left(a_{1} b_{2}+a_{2} b_{3}+a_{3} b_{1}-a_{2} b_{1}-a_{3} b_{2}-a_{1} b_{3}\right) E+\left(a_{2} a_{1} b_{2}+\right.$ $\left.a_{3} a_{2} b_{3}+a_{1} a_{3} b_{1}-a_{1} b_{1} a_{2}-a_{2} b_{2} a_{3}-a_{3} b_{3} a_{1}\right) E_{h}+\left(b_{1} a_{1} b_{2}+b_{2} a_{2} b_{3}+\right.$ $\left.b_{3} a_{3} b_{1}-b_{2} b_{1} a_{2}-b_{3} b_{2} a_{3}-b_{1} b_{3} a_{1}\right) E_{v}$. Proof in the case of Figure 19a:

Let us call $K=\left(a_{3}, b_{2}\right), L=\left(a_{1}, b_{2}\right), M=\left(a_{1}, b_{3}\right)$. Note that $\left(A_{1} A_{2} A_{3}\right)=\left(A_{2} L A_{1}\right)-\left(A_{3} M A_{1}\right)-\left(K L M A_{3}\right)-\left(A_{2} K A_{3}\right)$ The triangles and the quadrilateral on the right hand side of this equality are like those treated in Lemmas 13, 14. Using those formulas for their areas discharged from absolute values we can write (by taking care to use their positive orientations in the plane): 


$$
\begin{aligned}
\left(A_{1} A_{2} A_{3}\right)= & \left(A_{2} L A_{1}\right)-\left(A_{3} M A_{1}\right)-\left(K L M A_{3}\right)-\left(A_{2} K A_{3}\right) \\
= & \left(a_{2}-a_{1}\right)\left(b_{2}-b_{1}\right)\left(E+a_{1} E_{h}+b_{2} E_{v}\right) \\
& -\left(a_{3}-a_{1}\right)\left(b_{3}-b_{1}\right)\left(E+a_{1} E_{h}+b_{3} E_{v}\right) \\
& -\left(a_{3}-a_{1}\right)\left(b_{2}-b_{3}\right)\left[2 E+\left(a_{3}+a_{1}\right) E_{h}+\right. \\
& \left.+\left(b_{2}+b_{3}\right) E_{v}\right] \\
& -\left(a_{2}-a_{3}\right)\left(b_{2}-b_{3}\right)\left(E+a_{3} E_{h}+b_{2} E_{v}\right) \\
= & \left(a_{1} b_{2}+a_{2} b_{3}+a_{3} b_{1}-a_{2} b_{1}-a_{3} b_{2}-a_{1} b_{3}\right) E \\
& +\left(a_{2} a_{1} b_{2}+a_{3} a_{2} b_{3}+a_{1} a_{3} b_{1}-a_{1} b_{1} a_{2}-a_{2} b_{2} a_{3}\right. \\
& \left.-a_{3} b_{3} a_{1}\right) E_{h} \\
& +\left(b_{1} a_{1} b_{2}+b_{2} a_{2} b_{3}+b_{3} a_{3} b_{1}-b_{2} b_{1} a_{2}-b_{3} b_{2} a_{3}\right. \\
& \left.-b_{1} b_{3} a_{1}\right) E_{v}
\end{aligned}
$$

Proof in the case of Figure 19b: Let $K=\left(a_{2}, b_{1}\right), L=\left(a_{2}, b_{3}\right), M=$ $\left(a_{1}, b_{3}\right)$. Then (taking care about orientations as before):

$$
\begin{aligned}
\left(A_{1} A_{2} A_{3}\right)= & \left(A_{1} K L M\right)-\left(A_{1} K A_{2}\right)-\left(A_{2} L A_{3}\right)-\left(A_{1} M A_{3}\right) \\
= & \left(a_{2}-a_{1}\right)\left(b_{3}-b_{1}\right)\left(2 E+\left(a_{2}+a_{1}\right) E_{h}+\left(b_{3}+b_{1}\right) E_{v}\right) \\
& -\left(a_{2}-a_{1}\right)\left(b_{2}-b_{1}\right)\left(E+a_{2} E_{h}+b_{1} E_{v}\right) \\
& -\left(a_{2}-a_{3}\right)\left(b_{3}-b_{2}\right)\left(E+a_{2} E_{h}+b_{3} E_{v}\right) \\
& -\left(a_{3}-a_{1}\right)\left(b_{3}-b_{1}\right)\left(E+a_{1} E_{h}+b_{3} E_{v}\right) \\
= & \left(a_{1} b_{2}+a_{2} b_{3}+a_{3} b_{1}-a_{2} b_{1}-a_{3} b_{2}-a_{1} b_{3}\right) E \\
& +\left(a_{2} a_{1} b_{2}+a_{3} a_{2} b_{3}+a_{1} a_{3} b_{1}-a_{1} b_{1} a_{2}-a_{2} b_{2} a_{3}\right. \\
& \left.-a_{3} b_{3} a_{1}\right) E_{h} \\
& +\left(b_{1} a_{1} b_{2}+b_{2} a_{2} b_{3}+b_{3} a_{3} b_{1}-b_{2} b_{1} a_{2}-b_{3} b_{2} a_{3}\right. \\
& \left.-b_{1} b_{3} a_{1}\right) E_{v}
\end{aligned}
$$

as wanted.

Theorem 1. Let us consider a convex distorted lattice $L_{A B C D}\{m, n\}$ and let us assume without loss of generality that for the interior angles of $A B C D$ it is $\widehat{B}+\widehat{C} \leq 180^{\circ}, \widehat{C}+\widehat{D} \leq 180^{\circ}$. Let $T$ be a point such that $\overrightarrow{L_{10} T}=\overrightarrow{L_{00} L_{01}}$ and call $\left(L_{01} L_{00} L_{10}\right)=E,\left(L_{10} T L_{11}\right)=$ $E_{v},\left(L_{01} T L_{11}\right)=E_{h}$. If $A_{1} A_{2} \ldots A_{k}$ is a simple lattice polygon and $A_{i}=\left(a_{i}, b_{i}\right)=L_{a_{i} b_{1}}$ then 


$$
\begin{aligned}
& \pm\left(A_{1} A_{2} \ldots A_{k}\right)=\begin{array}{cccccccccc}
1 & 1 & 1 & 1 & \cdots & 1 & 1 & & & \\
& a_{1} & a_{2} & a_{3} & \cdots & a_{k-2} & a_{k-1} & a_{k} & a_{1} & E+ \\
& b_{1} & b_{2} & b_{3} & \cdots & b_{k-2} & b_{k-1} & b_{k} & b_{1} & E+ \\
1 & 1 & 1 & 1 & \ldots & 1 & 1 & & &
\end{array} \\
& \begin{array}{lllllll}
a_{2} & a_{3} & a_{4} & a_{5} & \cdots & a_{k} & a_{1}
\end{array}
\end{aligned}
$$

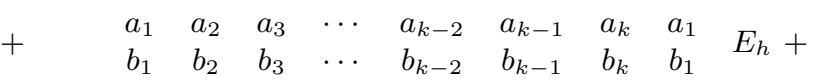

$$
\begin{aligned}
& \begin{array}{lllllll}
a_{1} & a_{2} & a_{3} & a_{4} & \cdots & a_{k-1} & a_{k}
\end{array} \\
& \begin{array}{lllllll}
b_{1} & b_{2} & b_{3} & b_{4} & \cdots & b_{k-1} & b_{k}
\end{array}
\end{aligned}
$$

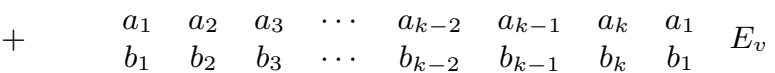

$$
\begin{aligned}
& \begin{array}{ccccccc}
b_{2} & b_{3} & b_{4} & b_{5} & \cdots & b_{k} & b_{1}
\end{array}
\end{aligned}
$$

The + sign holds whenever $\left(A_{1}, A_{2}, \cdots, A_{n}\right)$ is the positive orientation of $A_{1} A_{2} \ldots A_{k}$ in the plane.
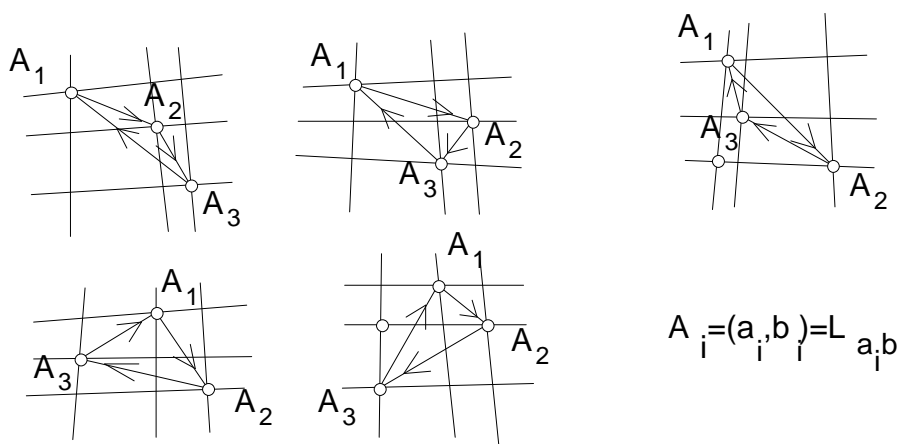

$$
A_{i}=\left(a_{i}, b_{i}\right)=L a_{i} b_{i}
$$
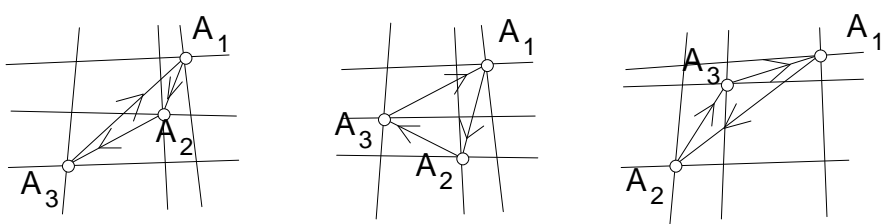

\section{FiguRE 18}

Note that because of Lemma 8 we can replace in any one of the above long determinants all indices simultaneously in all rows by the same cyclic permutation. 


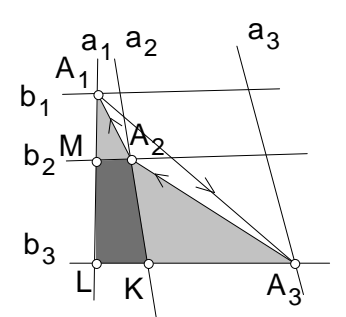

a

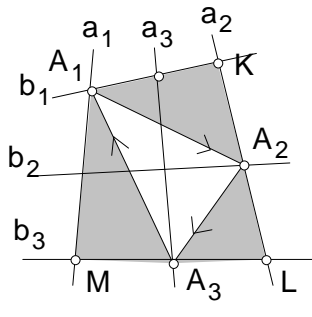

b

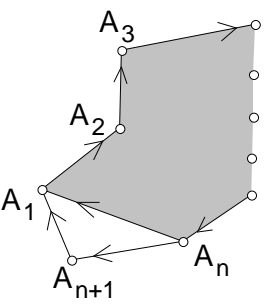

C

Figure 19

Proof. We prove it only in the case when $\left(A_{1}, A_{2}, \cdots, A_{n}\right)$ is the positive orientation of $A_{1} A_{2} \ldots A_{k}$ in the plane; the other case is proved similarly.

We shall develop an induction argument on $k$.

For $k=3$ the result was shown in the previous lemma. Let us assume the truth of the result for some value $k \geqq 3$.

Then for the value $k+1$ :

Let $A_{1} A_{2} \ldots A_{k} A_{k+1}$ be a lattice polygon and let us assume that $\left(A_{1}, A_{2}, \cdots, A_{k}, A_{k+1}\right)$ is its positive orientation in the plane.

It is $k+1 \geqq 4$ and by Lemma 1 there exists an internal diagonal with endpoints a pair of next to side vertices. In other words there exists an internal diagonal for which at least one of the two polygonal regions in which $\overline{A_{1} A_{2} \ldots A_{k} A_{k+1}}$ is partitioned is triangular. Without loss of generality we can assume that $A_{1} A_{k}$ is such a diagonal (Fig. 19c). Then $\left(A_{1} A_{2} \ldots A_{k} A_{k+1}\right)=\left(A_{1} A_{2} \ldots A_{k}\right)+\left(A_{1} A_{k} A_{k+1}\right)$.

Note that since $\left(A_{1}, A_{2}, \cdots, A_{k}, A_{k+1}\right)$ is the positive orientation of $A_{1} A_{2} \ldots A_{k} A_{k+1}$ in the plane, then the induced orientations $\left(A_{1}, A_{2}, \cdots, A_{k}\right),\left(A_{1}, A_{k}, A_{k+1}\right)$ are the positive orientations in the plane of the polygon $A_{1} A_{2} \ldots A_{k}$ and of the triangle $A_{1} A_{k} A_{k}$ respectively. Then by the induction hypothesis it holds:

$$
\left(A_{1} A_{2} \ldots A_{k}\right)=\left|\begin{array}{ccccccccc}
1 & 1 & 1 & 1 & \cdots & 1 & 1 & & \\
& a_{1} & a_{2} & a_{3} & \cdots & a_{k-2} & a_{k-1} & a_{k} & a_{1} \\
& b_{1} & b_{2} & b_{3} & \cdots & b_{k-2} & b_{k-1} & b_{k} & b_{1} \\
1 & 1 & 1 & 1 & \cdots & 1 & 1 & &
\end{array}\right| E+
$$




$$
\begin{aligned}
& +\left|\begin{array}{ccccccccc}
a_{2} & a_{3} & a_{4} & a_{5} & \cdots & a_{k} & a_{1} & & \\
& a_{1} & a_{2} & a_{3} & \cdots & a_{k-2} & a_{k-1} & a_{k} & a_{1} \\
& b_{1} & b_{2} & b_{3} & \cdots & b_{k-2} & b_{k-1} & b_{k} & b_{1} \\
a_{1} & a_{2} & a_{3} & a_{4} & \cdots & a_{k-1} & a_{k} & &
\end{array}\right| E_{h}+ \\
& +\left|\begin{array}{ccccccccc}
b_{1} & b_{2} & b_{3} & b_{4} & \cdots & b_{k-1} & b_{k} & & \\
& a_{1} & a_{2} & a_{3} & \cdots & a_{k-2} & a_{k-1} & a_{k} & a_{1} \\
& b_{1} & b_{2} & b_{3} & \cdots & b_{k-2} & b_{k-1} & b_{k} & b_{1} \\
b_{2} & b_{3} & b_{4} & b_{5} & \cdots & b_{k} & b_{1} & &
\end{array}\right| E_{v} \\
& =\left(\sum_{i=1}^{k-1} a_{i} b_{i+1}+a_{k} b_{1}-\sum_{i=1}^{k-1} b_{i} a_{i+1}-b_{k} a_{1}\right) E \\
& +\left(\sum_{i=1}^{k-1} a_{i+1} a_{i} b_{i}+a_{1} a_{k} b_{1}-\sum_{i=1}^{k-1} a_{i} b_{i} a_{i+1}-a_{k} b_{k} a_{1}\right) E_{h} \\
& +\left(\sum_{i=1}^{k-1} b_{i} a_{i} b_{i+1}+b_{k} a_{k} b_{1}-\sum_{i=1}^{k-1} b_{i+1} b_{i} a_{i+1}-b_{1} b_{k} a_{1}\right) E_{v}
\end{aligned}
$$

and

$$
\begin{aligned}
& \left(A_{1} A_{k} A_{k+1}\right)=
\end{aligned}
$$

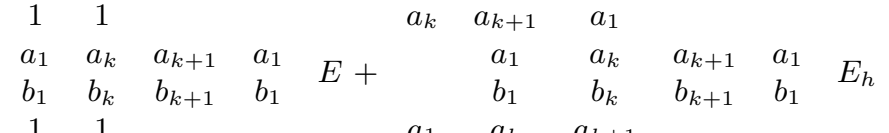

$$
\begin{aligned}
& \begin{array}{lll}
1 & 1 & 1
\end{array} \\
& a_{1} \quad a_{k} \quad a_{k+1} \\
& b_{1} \quad b_{k} \quad b_{k+1}
\end{aligned}
$$

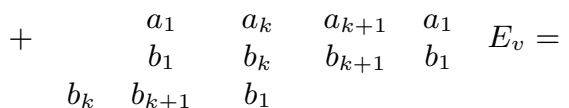

$$
\begin{aligned}
& =\left(a_{1} b_{k}+a_{k} b_{k+1}+a_{k+1} b_{1}-b_{1} a_{k}-b_{k} a_{k+1}-b_{k+1} a_{1}\right) E+\left(a_{k} a_{1} b_{k}\right. \\
& \left.+a_{k+1} a_{k} b_{k+1}+a_{1} a_{k+1} b_{1}-a_{1} b_{1} a_{k}-a_{k} b_{k} a_{k+1}-a_{k+1} b_{k+1} a_{1}\right) E_{h}+ \\
& \left(b_{1} a_{1} b_{k}+b_{k} a_{k} b_{k+1}+b_{k+1} a_{k+1} b_{1}-b_{k} b_{1} a_{k}-b_{k+1} b_{k} a_{k+1}-b_{1} b_{k+1} a_{1}\right) E_{v}
\end{aligned}
$$


So

$$
\begin{aligned}
& \left(A_{1} A_{2} \ldots A_{k} A_{k+1}\right) \\
= & \left(A_{1} A_{2} \ldots A_{k}\right)+\left(A_{k} A_{k-1} A_{k+1}\right) \\
= & \left(\sum_{i=1}^{k-1} a_{i} b_{i+1}+a_{k} b_{1}-\sum_{i=1}^{k-1} b_{i} a_{i+1}-b_{k} a_{1}+\right. \\
& \left.+a_{1} b_{k}+a_{k} b_{k+1}+a_{k+1} b_{1}-b_{1} a_{k}-b_{k} a_{k+1}-b_{k+1} a_{1}\right) E
\end{aligned}
$$


Corollary 2. Let us consider a convex distorted lattice $L_{A B C D}\{m, n\}$ for which $A B C D$ is a parallelogram (i.e., our lattice is a finite restriction of a regular infinite lattice), and let us call $\left(L_{01} L_{00} L_{10}\right)=$ $E$. If $A_{1} A_{2} \ldots A_{k}$ is a simple lattice polygon and $A_{i}=\left(a_{i}, b_{i}\right)$ then

$$
\text { (a) } \pm\left(A_{1} A_{2} \ldots A_{k}\right)=\frac{(A B C D)}{2 m n}\left|\begin{array}{ccccccccc}
1 & 1 & 1 & 1 & \ldots & 1 & 1 & & \\
& a_{1} & a_{2} & a_{3} & \cdots & a_{k-2} & a_{k-1} & a_{k} & a_{1} \\
& b_{1} & b_{2} & b_{3} & \cdots & b_{k-2} & b_{k-1} & b_{k} & b_{1} \\
1 & 1 & 1 & 1 & \cdots & 1 & 1 & &
\end{array}\right|
$$

(b) If moreover $L_{A B C D}\{m, n\}$ is part of the regular lattice of the integers in the plane, or more generally whenever the lattice quadrilaterals have unit area, the last formula becomes

$$
\pm\left(A_{1} A_{2} \ldots A_{k}\right)=\frac{1}{2}\left|\begin{array}{ccccccccc}
1 & 1 & 1 & 1 & \cdots & 1 & 1 & & \\
& a_{1} & a_{2} & a_{3} & \cdots & a_{k-2} & a_{k-1} & a_{k} & a_{1} \\
& b_{1} & b_{2} & b_{3} & \cdots & b_{k-2} & b_{k-1} & b_{k} & b_{1} \\
1 & 1 & 1 & 1 & \cdots & 1 & 1 & &
\end{array}\right|
$$

The + sign holds whenever $\left(A_{1}, A_{2}, \cdots, A_{n}\right)$ is the positive orientation of $A_{1} A_{2} \ldots A_{k}$ in the plane.

Proof. (a) The result comes immediately from the theorem since here we have $E_{h}=0=E_{v}$ and $E=\left(L_{00} L_{01} L_{10}\right)=\frac{\left(L_{00} L_{01} L_{11} L_{10}\right)}{2}=\frac{1}{2}$.

(b) The result comes from part (a) since here $(A B C D)=m n$. $($ area of any lattice quadrilateral $)=m n \cdot=m n$.

\section{Final Remarks}

The material presented so far is rich enough to deserve a few more comments. We organize these remarks roughly into sections.

4.1. About lattices. In the previous sections we dealt with lattices $L_{A B C D}\{m, n\}$ derived from polygons $A B C D$, mainly from convex ones. Unfortunately it is not true that all distorted lattices come from polygons. An example is given in Figure 4a where an $L\{4,1\}$ is depicted, whith $\left\{s_{1}, s_{2}\right\}$ say the horizontal segments and $\left\{l_{1}, l_{2}, l_{3}, l_{4}, l_{5}\right\}$ are the vertical ones.

Nevertheless the following conjecture might be true:

Conjecture 1. (a) If $L\{m, n\}$ is non trivial (i.e. $m \neq 1 \neq n$ ), then $L\{m, n\} \equiv L_{A B C D}\{m, n\}$ for some quadrilateral $A B C D$.

(b) For every $L\{m, n\}$ there exists a polygon (in the broad sense) $A_{1} A_{2} \cdots A_{k}$ whose sides lie on the lattice segments and such that 
all lattice segments lie in $\overline{A_{1} A_{2} \cdots A_{k}}$ partitioning this region into a number (mn maybe?) of quadrilaterals (in the broad sense).

For a quadrilateral $A B C D$ in the broad sense it is of course true that all segments joining two opposite sides lie in $\overline{A B C D}$ (Fig. 20a). Figure 20b shows a non-convex $A B C D$ for which the segments joining the midpoints of the opposite sides lie in $\overline{A B C D}$, while in Figure $20 \mathrm{c}$ these segments don't lie in $\overline{A B C D}$. Now note that Proposition 1

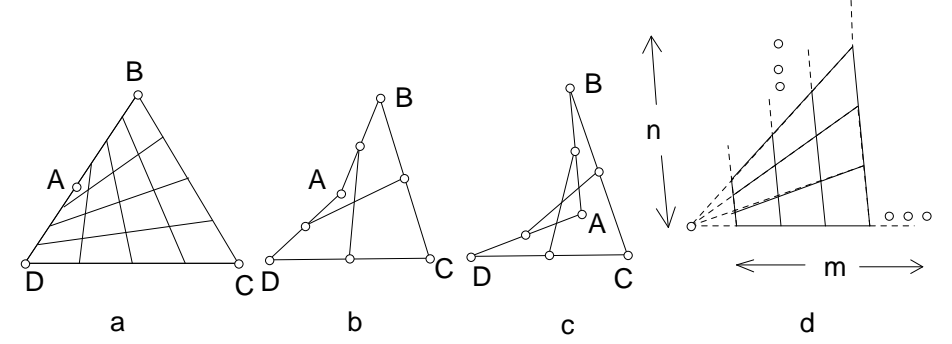

FigURE 20

holds also for $A B C D$ in the broad sense or even for a non-convex one, provided that all segments lie in $\overline{A B C D}$.

In other words the reader is invited to prove:

Proposition 2. Let $A B C D$ be a non-convex quadrilateral or one in a broad sense. Let us divide each of the sides $A B, D C$ in $m$ equal parts, and each of the sides $A D, B C$ in $n$ equal parts $\left(m, n \in \mathbb{N}^{*}\right)$ and join the corresponding division points of opposite sides by line segments. If all these segments lie in $\overline{A B C D}$ (as is the case when $A B C D$ is a quadrilateral in the broad sense) then each one of them from $A B$ to $D C$ is divided into $n$ equal parts, while each one from $A D$ to $B C$ is divided into $m$ equal parts.

Now comparing a general distorted lattice $L\{m, n\}$ to a usual (infinite) one, we can observe that unlike in the case of a usual lattice, the exist only finitely many ( $m$ or $n$ ) in number lattice sub-segments on each lattice line. Thus in order to hope for the possibility of simulating of situations of a usual lattice to a distorted one, we must first at least hope to prove that for any given pair of no matter how large natural numbers $m, n$, there exists a distorted $(m, n)$ lattice. Figure 20d shows how one can achieve this using Thales Theorem so 
that the vertical segments are parallel to each other, but the horizontal segments are not parallel to each other. Of course Proposition 1, answers on how to achieve this assuming that neither the horizontal or the vertical segments are parallel to each other.

Challenge 1. Generalize the notion of a distorted lattice into three or more dimensions.

\subsection{Corollaries of Proposition 1.}

Exercise 1. Let $A B C D$ be a convex quadrilateral and let $K, L, M, N$ be points on $A B, C D, A D, B C$ respectively such that $\frac{A K}{K B}=\frac{D L}{L C}=\kappa$, $\frac{A M}{M D}=\frac{B N}{N C}=\lambda$ with $\kappa, \lambda>0$. Then the intersection point $O$ of the segments $K L, M N$ divides the first in a ratio $\frac{K O}{O L}=\lambda$ and the second in a ratio $\frac{M O}{O N}=\kappa$. The result generalizes for points $K, L, M, N$ on the lines $A B, C D, A D, B C$ respectively.

Hint: First prove it for $\kappa, \lambda$ rational, say $\kappa=\frac{m_{1}}{m}, \lambda=\frac{n_{1}}{n}$ with the help of Proposition 1. Next, for arbitrary $\kappa, \lambda$ consider sequences of points $\left(K_{r} \in A K\right)_{r \in \mathbb{N}},\left(L_{r} \in D L\right)_{r \in \mathbb{N}},\left(M_{r} \in A M\right)_{r \in \mathbb{N}},\left(N_{r} \in\right.$ $B N)_{r \in \mathbb{N}}$, such that $\frac{A K_{r}}{K_{r} B}=\frac{D L_{r}}{L_{r} C}=\kappa_{r}$ and $\frac{A M_{r}}{M_{r} D}=\frac{B N_{r}}{N_{r} C}=\lambda_{r}$ with $\kappa_{r}, \lambda_{r}$ rational and $\kappa_{r} \stackrel{r \longrightarrow \infty}{\longrightarrow} \kappa, \lambda_{r} \stackrel{r \longrightarrow \infty}{\longrightarrow} \lambda$ (Fig. 21a).

Exercise 2. Let $A x, B y$ be half-lines on the same half-plane with respect to the line $A B$. If $A_{1}, A_{2}, \cdots$ are points on $A x$ and $B_{1}, B_{2}, \cdots$ points on $B y$ such that $A A_{1}=A_{1} A_{2}=\cdots$ and $B B_{1}=B_{1} B_{2}=\cdots$ then the midpoints of the segments $A B, A_{1} B_{1}, A_{2} B_{2}, \cdots$ all lie in the same line (Fig. 21b).

The proof comes immediately from Proposition 1.

One can try to prove a converse of Exercise 2:

Exercise 3. Let $\epsilon_{1}, \epsilon_{2}, \epsilon_{3}$ be lines in the plane, any two of them intersecting. Then for any $M_{2} \in \epsilon_{2}$, there exist unique $M_{1} \in \epsilon_{1}, M_{3} \in \epsilon_{3}$ such that $M_{2}$ is the midpoint of $M_{1} M_{3}$. Check what happens whenever it is not true that any two of $\epsilon_{1}, \epsilon_{2}, \epsilon_{3}$ intersect.

Hint: Call $O$ the intersection point of $\epsilon_{2}, \epsilon_{3}$ and consider the point $M^{\prime}$ such that $\overrightarrow{O M}=\overrightarrow{M M^{\prime}}$ (Fig. $\left.21 \mathrm{c}\right)$. Call $M_{1}$ the intersection of $\epsilon_{1}$ with the parallel to $\epsilon_{3}$ from $M^{\prime}$. Call $M_{3}$ the intersection of $\epsilon_{3}$ with the line $M_{1} M$. 


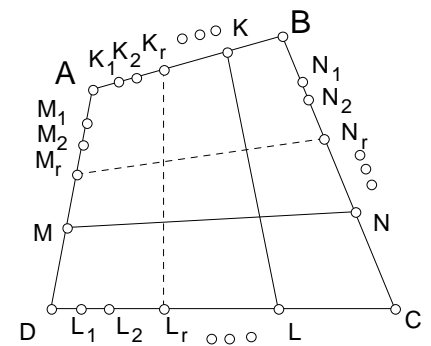

a

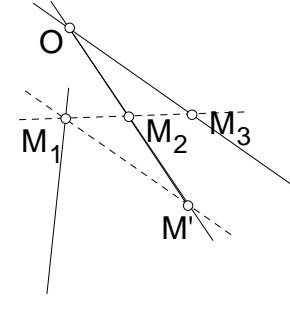

C

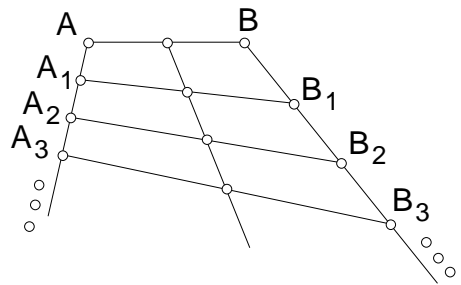

b

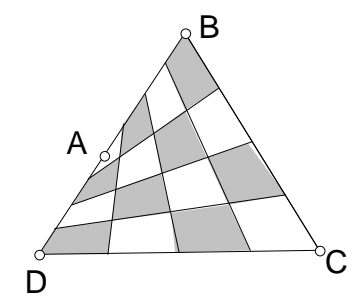

d

FiguRE 21

4.3. About areas. Proposition 2 can be used to generalize Corollary 1.

For example the following is true:

Exercise 4. Let $B C D$ be a triangle and $A$ be the midpoint of $B D$. We divide each one of the segments $A B, B C, C D, D A$ into four equal parts and we join the corresponding partition points of $A B, C D$ and of $B C, D A$. (Fig. 21c) Then $\overline{B C D}$ is partitioned into polygonal regions (all are quadrilateral except from a triangular one) which if coloured in a chess-like manner as black and white satisfy that the total black area equals the total white area.

Hint: Imitate the proof of Corollary 1 or proceed straight forward essentially applying a couple of times the proof of Lemma $7 \mathrm{~b}$.

The reader is invited to formulate a generalization of Corollary 1 .

Exercise 5. Prove Corollary 1 with the help of Theorem 1. 
Let us now move our attention from "total" (black or white) to "individual" polygonal areas in a lattice $L_{A B C D}\{m, n\}, A B C D$ convex. Recall from Section 1 that a potentially appropriate candidate for unit area substitute was decided there to be the set of the areas of the triangles $P_{1}=L_{00} L_{01} L_{11}, P_{2}=L_{01} L_{11} L_{10}, P_{3}=L_{00} L_{01} L_{10}$, $P_{4}=L_{00} L_{11} L_{10}$ in which the smallest (top left corner) quadrilateral $L_{00} L_{01} L_{11} L_{10}$ of the lattice is divided by its diagonals. Nevertheless the calculations were actually made in terms of only one of these areas $E=\left(L_{00} L_{01} L_{11}\right)$ and in terms of two other areas $E_{v}, E_{h}$. This "detachment" from our original plan occurred only for the shake of notational simplicity. According to the relations proved in Lemma 9 , the areas $\left(P_{1}\right),\left(P_{2}\right),\left(P_{3}\right),\left(P_{4}\right)$ are expressed in terms of $E, E_{v}, E_{h}$ and conversely these relations reveille that $E, E_{v}, E_{h}$ can be expressed in terms of $\left(P_{1}\right),\left(P_{2}\right),\left(P_{3}\right),\left(P_{4}\right)$.

Philosophically speaking, one can consider that in our area calculations $E$ is a "unit area"substitute, while $E_{v}, E_{h}$ express a "deficiency measure" for the distorted lattice $L_{A B C D}\{m, n\}$ to be a usual one (i.e., the restriction to a finite part of an infinite usual lattice in the plane).

By the way as is evident from Lemma 14 the areas of the quadrilaterals in which the distorted lattice $L_{A B C D}\{m, n\}$ ( $A B C D$ convex) is partitioned by its horizontal and vertical segments grow smaller as we move up or to the left. This makes the top left corner quadrilateral the one with the smallest possible area.

Challenge 2. If you succeeded in generalizing the notion of a distorted lattice into three or more dimensions, then generalize Proposition 1, Corollary 1, Theorem 1 in these dimensions as well.

Challenge 3. At least generalize Theorem 1 in higher dimensions in the case of the usual integer lattice $\mathbb{Z}^{n}, n \geqq 3$.

\subsection{About long determinants.}

Challenge 4. Provide a calculus of long determinants.

This calculus should contain properties referring to the relationship between long determinants and the usual determinants. One such property was given in Section 1.

The calculus should also contain "internal properties" of the long determinants like the one in the following 
Lemma 16. The following "gluing"property is true

$$
\begin{aligned}
& \left|\begin{array}{lllllll}
c_{1} & c_{2} & c_{3} & \cdots & c_{k-1} & & \\
& a_{1} & a_{2} & \cdots & a_{k-2} & a_{k-1} & a_{k} \\
& b_{1} & b_{2} & \cdots & b_{k-2} & b_{k-1} & b_{k} \\
d_{1} & d_{2} & d_{3} & \cdots & d_{k-1} & &
\end{array}\right|+ \\
& +\left|\begin{array}{ccccccc}
c_{k} & c_{k+1} & c_{k+2} & \cdots & c_{l-1} & & \\
& a_{k} & a_{k+1} & \cdots & a_{l-2} & a_{l-1} & a_{l} \\
& b_{k} & b_{k+1} & \cdots & b_{l-2} & b_{l-1} & b_{l} \\
d_{k} & d_{k+1} & d_{k+2} & \cdots & d_{l-1} & &
\end{array}\right|= \\
& =\left|\begin{array}{ccccccc}
c_{1} & c_{2} & c_{3} & \cdots & c_{l-1} & & \\
& a_{1} & a_{2} & \cdots & a_{l-2} & a_{l-1} & a_{l} \\
& b_{1} & b_{2} & \cdots & b_{l-2} & b_{l-1} & b_{l} \\
d_{1} & d_{2} & d_{3} & \cdots & d_{l-1} & &
\end{array}\right|
\end{aligned}
$$

Proof. By the definition of the long determinants, the formula to be proved is equivalent to

$$
\begin{gathered}
\left(\sum_{1}^{k-1} c_{i} a_{i} b_{i+1}-\sum_{1}^{k-1} d_{i} b_{i} a_{i+1}\right)+\left(\sum_{k}^{l-1} c_{i} a_{i} b_{i+1}-\sum_{k}^{l-1} d_{i} b_{i} a_{i+1}\right) \\
=\sum_{1}^{l-1} c_{i} a_{i} b_{i+1}-\sum_{1}^{l-1} d_{i} b_{i} a_{i+1}
\end{gathered}
$$

which in turn is equivalent to

$$
\begin{aligned}
\left(\sum_{1}^{k-1} c_{i} a_{i} b_{i+1}\right. & \left.+\sum_{k}^{l-1} c_{i} a_{i} b_{i+1}\right)-\left(\sum_{1}^{k-1} d_{i} b_{i} a_{i+1}+\sum_{k}^{l-1} d_{i} b_{i} a_{i+1}\right) \\
& =\sum_{1}^{l-1} c_{i} a_{i} b_{i+1}-\sum_{1}^{l-1} d_{i} b_{i} a_{i+1}
\end{aligned}
$$

which is of course true.

Challenge 5. Generalize the notion of the long determinant to include orthogonal arrays of more than 4 rows. Provide a calculus of these generalized long determinants.

Challenge 6. If you succeeded in intelligently generalizing the notion of the long determinant, find a "non trivial" geometric application of this generalization. 


\section{REFERENCES}

[1] R. Courant and H. Robbins, What is Mathematics?, Oxford University Press, 2nd ed. 1996.

[2] G. Martin, The Foundations of Geometry and the Non-Euclidean Plane, Springer Verlag, 3rd ed. 1996.

[3] S. Stein and S. Szabó, Algebra and Tiling: Homomorphisms in the Service of Geometry, MAA, 1994.

Dimitrios Kodokostas,

Technological Education Institute of Larissa,

Department of Computer Science,

41110 Larissa, Greece

dkodokostas@alumni.nd.edu,dkodokostas@teilar.gr

Received on 11 May 2003 and in final revised form on 16 March 2004. 